\title{
Article \\ Effects of Cranberry Juice Supplementation on Cardiovascular Disease Risk Factors in Adults with Elevated Blood Pressure: A Randomized Controlled Trial
}

\author{
Chesney K. Richter ${ }^{1}{ }^{\mathbb{D}}$, Ann C. Skulas-Ray ${ }^{1}$, Trent L. Gaugler ${ }^{2} \mathbb{D}$, Stacey Meily ${ }^{3}$, Kristina S. Petersen ${ }^{3,4} \mathbb{D}^{\mathbb{D}}$ \\ and Penny M. Kris-Etherton ${ }^{3, *}$
}

check for

updates

Citation: Richter, C.K.; Skulas-Ray, A.C.; Gaugler, T.L.; Meily, S.; Petersen, K.S.; Kris-Etherton, P.M. Effects of Cranberry Juice Supplementation on Cardiovascular Disease Risk Factors in Adults with Elevated Blood Pressure: A Randomized Controlled Trial. Nutrients 2021, 13, 2618. https://doi.org/10.3390/nu13082618

Academic Editors: Roberto Cangemi and Laura Chiavaroli

Received: 15 June 2021

Accepted: 24 July 2021

Published: 29 July 2021

Publisher's Note: MDPI stays neutral with regard to jurisdictional claims in published maps and institutional affiliations.

Copyright: (c) 2021 by the authors Licensee MDPI, Basel, Switzerland. This article is an open access article distributed under the terms and conditions of the Creative Commons Attribution (CC BY) license (https:/ / creativecommons.org/licenses/by/ $4.0 /)$.
1 Department of Nutritional Sciences, University of Arizona, Tucson, AZ 85716, USA; richterck@email.arizona.edu (C.K.R.); skulasray@email.arizona.edu (A.C.S.-R.)

2 Department of Mathematics, Lafayette College, Easton, PA 18042, USA; gauglert@lafayette.edu

3 Department of Nutritional Sciences, Pennsylvania State University, University Park, PA 16802, USA; sas117@psu.edu (S.M.); Kristina.Petersen@ttu.edu (K.S.P.)

4 Department of Nutritional Sciences, Texas Tech University, Lubbock, TX 79409, USA

* Correspondence: pmk3@psu.edu

\begin{abstract}
Emerging cardiovascular disease (CVD) risk factors, including central vascular function and HDL efflux, may be modifiable with food-based interventions such as cranberry juice. A randomized, placebo-controlled, crossover trial was conducted in middle-aged adults with overweight/obesity ( $n=40$; mean BMI: $28.7 \pm 0.8 \mathrm{~kg} / \mathrm{m}^{2}$; mean age: $47 \pm 2$ years) and elevated brachial blood pressure (mean systolic/diastolic BP: $124 \pm 2 / 81 \pm 1 \mathrm{~mm} \mathrm{Hg}$ ). Study participants consumed $500 \mathrm{~mL} / \mathrm{d}$ of cranberry juice ( $16 \mathrm{fl} \mathrm{oz;} 27 \%$ cranberry juice) or a matched placebo juice in a randomized order (8-week supplementation periods; 8-week compliance break), with blood samples and vascular measurements obtained at study entry and following each supplementation period. There was no significant treatment effect of cranberry juice supplementation on the primary endpoint of central systolic blood pressure or central or brachial diastolic pressure. Cranberry juice significantly reduced 24-h diastolic ambulatory BP by $\sim 2 \mathrm{~mm} \mathrm{Hg}$ compared to the placebo $(p=0.05)$ during daytime hours. Cranberry juice supplementation did not alter LDL-C but significantly changed the composition of the lipoprotein profile compared to the placebo, increasing the concentration of large LDL-C particles (+29.5 vs. $-6.7 \mathrm{nmol} / \mathrm{L} ; p=0.02)$ and LDL size (+0.073 vs. $-0.068 \mathrm{~nm} ; p=0.001)$. There was no effect of treatment on ex vivo HDL efflux in the total population, but exploratory subgroup analyses identified an interaction between BMI and global HDL efflux ( $p=0.02)$, with greater effect of cranberry juice in participants who were overweight. Exploratory analyses indicate that baseline C-reactive protein (CRP) values may moderate treatment effects. In this population of adults with elevated blood pressure, cranberry juice supplementation had no significant effect on central systolic blood pressure but did have modest effects on 24-h diastolic ambulatory BP and the lipoprotein profile. Future studies are needed to verify these findings and the results of our exploratory analyses related to baseline health moderators.
\end{abstract}

Keywords: lipids; LDL-C; blood pressure; arterial stiffness; inflammatory markers

\section{Introduction}

Cardiovascular disease (CVD) and related co-morbidities remain the leading cause of death worldwide [1,2]. Current dietary guidelines for reducing CVD risk recommend dietary patterns that emphasize bioactive-rich, plant-based foods [3,4]. Cranberries have a unique phytochemical profile and are a particularly good source of proanthocyanins, anthocyanins, flavonoids, and phenolic acids [5,6]. In vitro studies have shown that cranberry polyphenols exert strong anti-inflammatory and antioxidant properties [5,7]. However, this does not always translate directly to significant clinical effects, and additional re- 
search is needed to identify which CVD risk factors may be modifiable with cranberry juice supplementation.

Previous studies of cranberry supplementation have placed little emphasis on blood pressure and vascular function outcomes. A recent meta-analysis of cranberry supplementation studies found no effect on diastolic blood pressure, but systolic blood pressure was significantly lowered $(-4 \mathrm{~mm} \mathrm{Hg})$, particularly in participants over 50 years old [8]. Beyond brachial blood pressure, central blood pressure and indices of arterial stiffness are more accurate predictors of cardiovascular events and disease severity $[9,10]$. To our knowledge, only two previous cranberry supplementation studies have investigated these endpoints. Low-calorie cranberry juice had no effect on augmentation index (AI) in overweight men compared to the control [11], while double-strength cranberry juice (54\% juice) significantly reduced pulse wave velocity (PWV) in patients with stable coronary artery disease [12].

Cranberry bioactives may also be beneficial for atherogenic lipoproteins. For instance, cranberry juice has been shown to significantly increase high-density lipoproteincholesterol (HDL-C) in adults with abdominal obesity [13], reduce triglycerides in healthy adults [14], and reduce apolipoprotein B while increasing apolipoprotein A-I in patients with type 2 diabetes [15]; however, these effects were not observed in other studies of cranberry juice [16-18] and cranberry extracts [19]. In a recent meta-analysis of studies using a variety of different types of cranberry supplements $(n=10)$, there was a significant increase in HDL-C, particularly for participants less than 50 years of age, but there was no effect on total cholesterol, LDL-C, or triglycerides [8]. HDL-C efflux capacity may also be a more accurate measure of CVD risk than HDL-C concentration [20] and warrants investigation related to cranberry supplementation.

The present study was designed to investigate whether daily consumption of cranberry juice ( $16 \mathrm{fl} \mathrm{oz}$ per day) for $8-12$ weeks could improve specified CVD risk factors in adults with elevated blood pressure. We hypothesized that cranberry juice would significantly improve vascular function, reduce atherogenic lipoproteins, and improve HDL function.

\section{Materials and Methods}

\subsection{Study Population}

Men and women who were 30-65 years of age, had elevated resting blood pressure (systolic blood pressure $\geq 120 \mathrm{~mm} \mathrm{Hg}$ and/or diastolic blood pressure $\geq 80 \mathrm{~mm} \mathrm{Hg}$ and $<160 / 100 \mathrm{~mm} \mathrm{Hg}$ ), had a BMI of $\geq 18$ and $\leq 39 \mathrm{~kg} / \mathrm{m}^{2}$, and otherwise free of any serious illness were recruited for the study. Other inclusion criteria included fasting TG $(<350 \mathrm{mg} / \mathrm{dL})$ and total cholesterol ( $<284 \mathrm{mg} / \mathrm{dL}$ for women and $<273 \mathrm{mg} / \mathrm{dL}$ for men). Exclusion criteria included smoking and/or use of other tobacco products; a history of diabetes, autoimmune disorders, and heart, liver, kidney, or uncontrolled thyroid disease; pregnancy, lactation, or a desire to become pregnant during the study; and chronic use of non-steroidal anti-inflammatories or medications/supplements for elevated lipids, blood pressure, or glucose.

\subsection{Participant Recruitment}

Participants were recruited and consented for the study from December 2015 to October 2018. Recruitment activities included fliers in the community, campus e-mail lists, local newspaper advertisements, and a university research website. Potential subjects emailed or called to indicate interest in participating and were then given additional information about the study. If interested, they were asked a series of medical history and lifestyle questions to screen for eligibility. After written informed consent was obtained, a urine pregnancy test was performed for women of child-bearing potential, and blood pressure was measured according to JNC 7 Guidelines [21]. Briefly, after a 5-min seated rest, three readings were taken by nurses in a controlled environment using a calibrated mercury sphygmomanometer. The mean of the last 2 readings was used to determine eligibility. If an individual's blood pressure met the study inclusion criteria, body weight and height 
were measured (without shoes and in light clothing) to calculate BMI. A blood sample was then drawn for a complete blood count and standard chemistry profile (lipid panel, glucose, liver, and kidney function) to rule out the presence of illness (autoimmune disease, cancer, and immunodeficiency). A balanced randomization scheme with a block size of four was developed in advance (by Dr. Ann Skulas-Ray) using an online randomization generator, and subjects were assigned to a treatment sequence at enrollment (by Danette Teeter and Stacey Meily). Sample size was determined based on a power calculation, with central systolic blood pressure as the primary outcome. Based on variability of central systolic blood pressure in our previous work [22], it was estimated that 34 participants would provide $80 \%$ power to detect a $4.5 \mathrm{~mm} \mathrm{Hg}$ change with a significance level of 0.05 . This study was conducted according to the guidelines issued in the Declaration of Helsinki, and all procedures involving human subjects were approved by the Institutional Review Board of the Pennsylvania State University. Written informed consent was obtained from all participants. The study is registered at clinicaltrials.gov as NCT02556749.

\subsection{Study Design and Intervention}

This was a randomized, placebo-controlled, 2-period crossover study with 8-week treatment periods separated by an approximate 8-week compliance break. Treatment periods were extended by up to 4 weeks ( 12 weeks total) in the case of illness, injury, or scheduling difficulties. During the treatment periods, participants received $500 \mathrm{~mL} / \mathrm{d}$ ( $16 \mathrm{fl} \mathrm{oz}$ ) of cranberry juice ( $\sim 70 \mathrm{kcal} ; 27 \%$ cranberry juice) or $500 \mathrm{~mL} / \mathrm{d}(\sim 16 \mathrm{fl} \mathrm{oz})$ of a placebo juice (matched for color, calorie, and carbohydrates), in random order. Additional information regarding the nutrient profile, bioactive content, and ingredients of the study juices can be found in Tables S1 and S2. The placebo beverage was formulated to match appearance, aroma, and taste of the cranberry juice and to have a similar amount of total calories, total carbohydrates, and total organic acids. Treatments consisted of individually packaged juice provided in identical bottles during both periods. Treatments were matched to coded alphanumeric identifiers so that the investigators and participants were blinded to treatment assignment. The juices were matched for taste and appearance to maintain the blinding of participants and investigators to treatment sequence. All juices were formulated and provided by Ocean Spray (Middleborough, MA, USA).

Participants were instructed to consume two bottles of juice per day during each treatment period, either all at once or divided throughout the day (e.g., $8 \mathrm{fl} \mathrm{oz}$ with breakfast and $8 \mathrm{fl} \mathrm{oz}$ with dinner). They were provided with two weeks' worth of juice at a time and were asked to incorporate the study juices into their habitual diet, maintaining their normal dietary intake and physical activity. In addition, participants were instructed to avoid consuming any other food or drinks that contained cranberry or cranberry juice for the duration of the study. Compliance with the study interventions was assessed via self-report. Participants completed a daily consumption log to track their consumption of the study juice each day. Participants reported to the Clinical Research Center on a biweekly basis. At this time, a member of the research team who was not involved in the statistical analyses provided participants with additional study juice and reviewed their body weight and juice consumption logs.

Blood sampling and vascular testing were performed at baseline and following each 8-week supplementation period. All study procedures were conducted at the Pennsylvania State University CRC according to standardized protocols. During the $48 \mathrm{~h}$ prior to testing visits, participants were instructed to avoid alcohol; refrain from taking non-approved medications and supplements, including pain relievers, vitamins, or minerals; and limit their intake of coffee and tea to no more than 1 standard beverage per day. Strenuous exercise was avoided for $12 \mathrm{~h}$ prior to testing. Testing visits were conducted following an overnight fast (no food or drink other than water for $12 \mathrm{~h}$ ). Endpoint measurements were conducted on two consecutive days. On one day, vascular function testing was performed prior to blood sampling. On the other day of testing, only a fasting blood draw was performed. Pre-menopausal women were scheduled for vascular testing within the first 
7 days of starting their menstrual period in order to minimize hormonal effects on vascular endpoints. Ambulatory blood pressure was also measured at baseline and the end of each supplementation period, with the device worn for a 24-h period on each occasion. Typically, participants were fitted with the device on the first day of testing and returned it on the following testing day. All study procedures were completed in May 2019. Participants received monetary compensation of $\$ 200$ for completion of the study.

\subsection{Vascular Function Measures}

Central blood pressure and arterial stiffness indices were assessed using the SphygmoCor XCEL System pulse waveform analysis (AtCor Medical, Sydney, Australia). All measurements were performed in a temperature-controlled, quiet, dimly lit room.

\subsection{Pulse Wave Analysis (PWA): Central (Aortic) Blood Pressure and Augmentation Index (AI)}

Following a 5-min seated rest, central pressures and wave reflection characteristics (i.e., augmentation pressure (AP) and $\mathrm{AI}$ ) were derived from brachial pressure waveforms using a generalized transfer function that is considered to be substantially equivalent to generalized transfer functions for radial tonometry validated against an indwelling catheter [23-25]. At each visit, three PWA measurements were taken, following JNC 7 Blood Pressure Guidelines [21], with 1 min between each reading. The last two PWA results were averaged and used for analysis. The AI was standardized to a heart rate of 75 beats per minute (AI@75) to correct for the independent inverse effect of heart rate on augmentation of the pulse wave form [26].

\subsection{Pulse Wave Velocity (PWV)}

Aortic stiffness was assessed by carotid-femoral pulse wave velocity (PWV). Carotid and femoral arterial pressure waveforms were measured simultaneously via an applanation tonometry sensor manually held in place above the right common carotid artery and a blood pressure cuff placed on the right femoral artery. Distance measurements were taken from the sternal notch to the carotid artery, from the sternal notch to the top of the femoral cuff and from the femoral artery to the top of the femoral cuff. Based on these measurements, the SphygmoCor XCEL System automatically calculates the distance traveled by the pulse wave from the carotid artery to the femoral artery. Transit time between the carotid and femoral pressure waves is determined by the SphygmoCor System using the foot-to-foot method [27]. PWV is then calculated as distance over transit time. At each visit, three PWV measurements were obtained in the supine position, with $1 \mathrm{~min}$ between readings. The last two PWV results were averaged for analysis.

\subsection{Ambulatory Blood Pressure Monitoring}

Participants wore an ambulatory blood pressure monitor for a 24-h period at baseline and the end of each treatment period. Following baseline day 1 testing and during the last week of each treatment period, the monitor (Mortara Instrument Inc., Milwaukee, WI) was fitted by study personnel on the non-dominant arm and programmed to automatically capture a reading every $20 \mathrm{~min}$ during the day and every $30 \mathrm{~min}$ overnight (10 p.m. to 6 a.m.). The mean number of successful readings obtained in a 24-h period was $50 \pm 10$, with $36 \pm 8$ awake readings and $13 \pm 5$ asleep readings.

\subsection{Blood Sample Collection and Assay Methods}

Blood drawn into anticoagulant-coated tubes containing lithium heparin or EDTA was immediately centrifuged for $15 \mathrm{~min}$ at $1500 \times \mathrm{g}$. Blood drawn into serum separator tubes was allowed to clot for $30 \mathrm{~min}$ prior to centrifugation. Total cholesterol and triglycerides (TG) were measured by enzymatic procedures (Quest Diagnostics, Pittsburgh, PA, USA; $\mathrm{CV}<2 \%$ for both). HDL-C was estimated according to the modified heparin-manganese procedure (Quest Diagnostics; CV $<2 \%$ ). LDL-C was measured directly via enzymatic analysis. Glucose was determined by Spectrophotometry procedures (Quest Diagnostics). 
Insulin was measured by radioimmunoassay using ${ }^{125} \mathrm{I}$-labeled human insulin and a human insulin antiserum (Quest Diagnostics). Serum high-sensitivity C-reactive protein (CRP) was measured by latex-enhanced immunonephelometry (Quest Diagnostics; assay $\mathrm{CV}<8 \%$ ). For other endpoints, aliquots of serum and plasma were immediately stored at $-80{ }^{\circ} \mathrm{C}$ for batch analysis. Plasma lipoprotein particle number and size were assessed by a proton magnetic resonance spectroscopy assay (NMR LipoProfile III; LipoScience, Raleigh, NC, USA), which measures the particle concentrations of lipoprotein subclasses and mean particle size of lipoproteins. Plasma isoprostane concentrations were measured by the Vanderbilt University Eicosanoid Core Laboratory using gas chromatography/negative-ion chemical ionization mass spectrometry (GC/NICI-MS), as described previously [28].

\subsection{Cholesterol Efflux}

Serum HDL (apoB-depleted serum) was prepared from individual serum samples by precipitation of apoB-containing lipoproteins using polyethylene glycol (PEG). Briefly, for each serum sample, 100 parts serum is mixed with 40 parts PEG $(20 \%, v / v$, in glycine buffer, $\mathrm{pH}$ 7.4). The mixture is incubated at room temperature for $20 \mathrm{~min}$ and then centrifuged at 10,000 rpm for $30 \mathrm{~min}$ at $4{ }^{\circ} \mathrm{C}$. The supernatant containing serum HDL is collected and used for analysis of cholesterol efflux capacity. Cholesterol efflux capacities of serum HDL samples were determined as described in detail elsewhere $[29,30]$. In brief, global and ABCA1-mediated cholesterol efflux were measured using J774 mouse macrophage cells in the presence and/or absence of cAMP. For all assays, cells were pre-incubated with $\left[{ }^{3} \mathrm{H}\right]$-cholesterol and ACAT inhibitor Sandoz 58-035 (but not preloaded with mass cholesterol) overnight. Cells were then incubated overnight in $0.2 \%$ BSA with or without cpt-cAMP. After washing, the cells were incubated for $4 \mathrm{~h}$ with the serum HDL samples (apoB-depleted serum) added at $2.8 \%(v / v) .\left[{ }^{3} \mathrm{H}\right]$-cholesterol released to serum after incubation with cells for $4 \mathrm{~h}$ was measured by liquid scintillation counting. Cholesterol efflux is expressed as the radiolabel released as a percentage of $\left[{ }^{3} \mathrm{H}\right]$-cholesterol within cells before addition of serum. All efflux values were corrected by subtracting the small amount of radioactive cholesterol released from cells incubated with serum-free medium. The global cholesterol efflux from J774 cells treated with cAMP includes cholesterol efflux mediated by ABCA1, SR-BI, ABCG1, passive diffusion, or other still unknown carriers [30]. ABCA1-dependent efflux from J774 cells was determined as the difference in efflux from cAMP-treated and untreated cells. Cholesterol efflux from untreated cells is mediated by SR-BI, ABCG1, passive diffusion, or other still unknown carriers.

\subsection{Statistical Analyses}

All statistical analyses were performed using SAS (version 9.4; SAS Institute). Only participants who completed both supplementation periods were included in analyses. Participants with CRP values $\geq 10 \mathrm{mg} / \mathrm{L}(n=4)$ were excluded from analyses of CRP, as these values are indicative of acute inflammation from an isolated physical injury and/or infection [31]. Differences between male and female participants at baseline were assessed via independent two-sample $t$-test (PROC TTEST). Change scores for end-oftreatment values were calculated by subtracting study-entry baseline values from each post-supplementation measure. For end-of-treatment values, the mixed models procedure (PROC MIXED) in SAS was used to test the effects of treatment, period, and treatment by period interactions for each outcome. Outcome variables were assessed for normality (PROC UNIVARIATE). Baseline values were included as covariates for each endpoint. Outcomes were modeled as repeated measures, with subject as a random effect and with unstructured variance for treatment/period. Treatment by period interactions were included in order to test for potential carry-over effects between intervention periods. If significant, the treatment by period interaction was retained in the model, and lower order effects of treatment and period were not interpreted. When period and treatment by period interactions were nonsignificant, they were removed from the model. When period effects were significant, they were retained in the final model of treatment effects. Values that were 
measured in duplicate on separate days (i.e., body weight and lipids) were averaged prior to analysis. Subgroup analyses were conducted for measures of HDL efflux to investigate the influence of BMI on treatment effects. Participants were categorized as normal weight (BMI: $18.5-24.9 \mathrm{~kg} / \mathrm{m}^{2}$ ), overweight (BMI: $25-29.9 \mathrm{~kg} / \mathrm{m}^{2}$ ), or obese (BMI: $\geq 30 \mathrm{~kg} / \mathrm{m}^{2}$ ). An exploratory analysis was also conducted to investigate the effect of baseline CRP on treatment effects. Participants were split into high and low CRP categories based on the median baseline CRP value of $1.3 \mathrm{mg} / \mathrm{L}$. Means are reported as least-squares means $\pm \mathrm{SEM}$ (standard error of the mean). For all tests, $\alpha$ was set at 0.05 .

\section{Results}

A schematic of participant recruitment for the study is provided in Figure 1. Of the 119 individuals who were screened, 47 met eligibility criteria and were enrolled in the study. Seven participants withdrew during the study due to unrelated health concerns $(n=2)$, inability to obtain study measurements $(n=2)$, and noncompliance with study protocol $(n=3)$. Thus, data are reported for 40 participants. Based on completion of self-reported daily consumption logs, compliance with the study protocol was $90 \%$.

The screening characteristics of participants who completed the study are presented in Table 1. At baseline, there was a significant difference between female $(n=15)$ and male $(n=25)$ participants for select blood pressure and lipid/lipoprotein measurements. Female participants were significantly older ( $53 \pm 11$ years vs. $43.5 \pm 11$ years; $p=0.01$ ) and had higher HDL-C ( $56 \pm 11 \mathrm{mg} / \mathrm{dL}$ vs. $44 \pm 10 \mathrm{mg} / \mathrm{dL} ; p=0.002)$, central systolic blood pressure $(119 \pm 12 \mathrm{~mm} \mathrm{Hg}$ vs. $112 \pm 7 \mathrm{~mm} \mathrm{Hg} ; p=0.05)$, pulse pressure $(39 \pm 7.5 \mathrm{~mm} \mathrm{Hg}$ vs. $30 \pm 4 \mathrm{~mm} \mathrm{Hg} ; p=0.0004)$, augmentation pressure $(13.3 \pm 5.2 \mathrm{~mm} \mathrm{Hg}$ vs. $6.1 \pm 3.1 \mathrm{~mm} \mathrm{Hg}$; $p<0.0001)$, and augmentation index (30.8 $\pm 23.3 \%$ vs. $16.5 \pm 12.2 \% ; p=0.0005)$ values. Men had significantly higher triglycerides (122 $\pm 48 \mathrm{mg} / \mathrm{dL}$ vs. $90 \pm 17 \mathrm{mg} / \mathrm{dL} ; p=0.005)$. Significant treatment by sex interactions were found for total cholesterol (interaction $p=0.04$ ), medium HDL particles (interaction $p=0.007$ ), and small HDL particles (interaction $p=0.02$ ) (Figure S1). There was no significant effect of supplementation on weight. Baseline values according to treatment randomization order are also presented in Table 1 . There were no significant differences according to treatment order for any variables $(p>0.05)$.

Table 1. Screening characteristics of participants who completed the study $(n=40 ; 25 \mathrm{M}, 15 \mathrm{~F})$ and baseline characteristics of participants according to treatment randomization order ${ }^{1}$.

\begin{tabular}{cccc}
\hline & Screening Visit & \multicolumn{2}{c}{ Baseline Visit } \\
\hline Age $(\mathrm{y})$ & Pooled & Cranberry-Placebo Sequence & Placebo-Cranberry Sequence \\
\hline BMI $\left(\mathrm{kg} / \mathrm{m}^{2}\right)$ & $47 \pm 12(30-64)$ & $43 \pm 12$ & $50 \pm 10$ \\
\hline Normal weight $\left(18.5-24.9 \mathrm{~kg} / \mathrm{m}^{2}\right)$ & $28.8 \pm 4.7(22.2-39.1)$ & $28.6 \pm 4.6$ & $n=4.9 \pm 4.9$ \\
\hline Overweight $\left(25-29.9 \mathrm{~kg} / \mathrm{m}^{2}\right)$ & $n=9$ & $n=8$ & $n=8$ \\
\hline Obese $\left(\geq 30 \mathrm{~kg} / \mathrm{m}^{2}\right)$ & $n=16$ & $n=6$ & $n=9$ \\
\hline Systolic blood pressure $(\mathrm{mm} \mathrm{Hg})$ & $124 \pm 9(108-139)$ & $126 \pm 6$ & $123 \pm 13$ \\
\hline Diastolic blood pressure $(\mathrm{mm} \mathrm{Hg})$ & $85 \pm 6(72-97)$ & $82 \pm 6$ & $79 \pm 8$ \\
\hline Glucose $(\mathrm{mg} / \mathrm{dL})$ & $94 \pm 8(79-113)$ & $91 \pm 7$ & $93 \pm 7$ \\
\hline Total cholesterol $(\mathrm{mg} / \mathrm{dL})$ & $195 \pm 31(121-268)$ & $184 \pm 32$ & $200 \pm 42$ \\
\hline HDL-C $(\mathrm{mg} / \mathrm{dL})$ & $49 \pm 13(30-84)$ & $45 \pm 11$ & $51 \pm 12$ \\
\hline TC:HDL-C & $4.2 \pm 1.2(1.8-7.8)$ & $4.3 \pm 1.0$ & $4.1 \pm 1.3$ \\
\hline LDL-C $(\mathrm{mg} / \mathrm{dL})$ & $124 \pm 28(54-176)$ & $115 \pm 27$ & $128 \pm 39$ \\
\hline Triglycerides $(\mathrm{mg} / \mathrm{dL})$ & $112 \pm 46(51-230)$ & $123 \pm 47$ & $99 \pm 34$ \\
\hline
\end{tabular}

${ }^{1}$ Values are means $\pm \mathrm{SD}(n=40 ; 25 \mathrm{M}, 15 \mathrm{~F})$ with ranges in parentheses and were calculated using the univariate procedure (SAS version 9.4; SAS Institute Inc., Cary, NC, USA). There were no significant baseline differences for any values by treatment order $(p>0.05)$. Abbreviations: BMI, body mass index; HDL-C, high-density lipoprotein-cholesterol; LDL-C, low-density lipoprotein-cholesterol; TC, total cholesterol. 


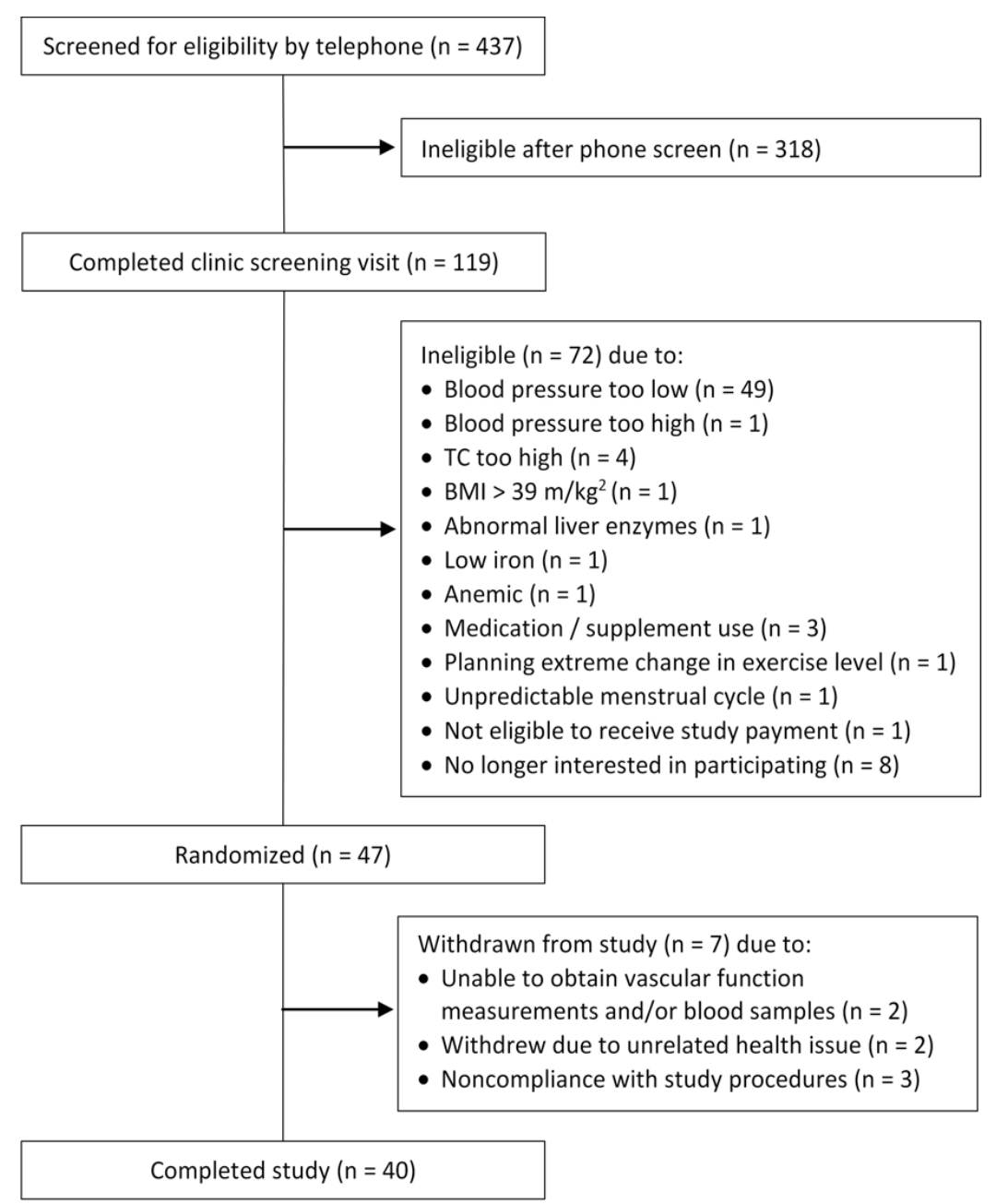

Figure 1. Schematic of participant recruitment and exclusion.

\subsection{Effect of Cranberry Juice on Measures of Vascular Function}

There were no significant main effects of treatment for the primary endpoint of central systolic blood pressure, central or brachial diastolic blood pressure, nor for any other measures of brachial or central blood pressure, augmentation index, and pulse wave velocity (Table 2). Cranberry supplementation significantly reduced ambulatory diastolic blood pressure compared to the placebo (mean difference $=-1.79 \pm 0.9 \mathrm{~mm} \mathrm{Hg} ; p=0.05$ ), with the change occurring during awake, daytime hours (mean difference $=2.0 \pm 1.0 \mathrm{~mm}$ $\mathrm{Hg} ; p=0.05)$ and not during the nighttime period $(p>0.9)$ (Figure 2). Cranberry juice did not alter any other ambulatory blood pressure values.

Table 2. Vascular function and arterial stiffness values in study participants $(n=40)$ following each 8-week supplementation period ${ }^{1}$.

\begin{tabular}{|c|c|c|c|c|c|}
\hline & Baseline & Cranberry & Placebo & Treatment $p$-Value & Period $p$-Value \\
\hline Brachial SBP (mm Hg) & $124 \pm 1$ & $123 \pm 1$ & $125 \pm 1$ & 0.1 & 0.2 \\
\hline Brachial DBP (mm Hg) & $81 \pm 1$ & $79 \pm 1$ & $81 \pm 1$ & 0.3 & 0.3 \\
\hline Central SBP (mm Hg) & $115 \pm 1$ & $113 \pm 1$ & $115 \pm 1$ & 0.2 & 0.2 \\
\hline Central DBP (mm Hg) & $81 \pm 1$ & $80 \pm 1$ & $81 \pm 1$ & 0.3 & 0.3 \\
\hline Central PP (mm Hg) & $33 \pm 1$ & $33 \pm 1$ & $34 \pm 1$ & 0.5 & 0.5 \\
\hline
\end{tabular}


Table 2. Cont.

\begin{tabular}{|c|c|c|c|c|c|}
\hline & Baseline & Cranberry & Placebo & Treatment $p$-Value & Period $p$-Value \\
\hline Augmentation Pressure (mm Hg) & $8.8 \pm 0.6$ & $8.5 \pm 0.5$ & $8.4 \pm 0.4$ & 0.8 & 0.3 \\
\hline Augmentation Index & $21.9 \pm 1.5$ & $20.7 \pm 1.0$ & $20.7 \pm 1.0$ & $>0.9$ & 0.3 \\
\hline Pulse Wave Velocity (m/s) & $7.0 \pm 0.1$ & $7.1 \pm 0.1$ & $7.1 \pm 0.1$ & 0.6 & 0.4 \\
\hline 24-h SBP (mm Hg) & $124 \pm 1$ & $123 \pm 1$ & $124 \pm 1$ & 0.4 & 0.3 \\
\hline 24-h DBP (mm Hg) & $79 \pm 1$ & $78 \pm 1$ & $80 \pm 1$ & 0.05 & 0.4 \\
\hline 24-h PP (mm Hg) & $70 \pm 1$ & $71 \pm 1$ & $70 \pm 1$ & 0.3 & 0.1 \\
\hline Daytime SBP (mm Hg) & $129 \pm 1$ & $128 \pm 1$ & $129 \pm 1$ & 0.4 & 0.2 \\
\hline Daytime DBP (mm Hg) & $82 \pm 1$ & $81 \pm 1$ & $83 \pm 1$ & 0.05 & 0.3 \\
\hline Daytime PP (mm Hg) * & $73 \pm 1$ & $73 \pm 1$ & $72 \pm 1$ & 0.3 & 0.05 \\
\hline Nighttime SBP (mm Hg) & $112 \pm 2$ & $111 \pm 1$ & $109 \pm 2$ & 0.4 & 0.5 \\
\hline Nighttime DBP (mm Hg) & $72 \pm 1$ & $71 \pm 1$ & $71 \pm 1$ & $>0.9$ & 0.8 \\
\hline Nighttime PP (mm Hg) & $63 \pm 1$ & $64 \pm 1$ & $64 \pm 1$ & 0.7 & 0.9 \\
\hline
\end{tabular}

${ }^{1}$ All values are means \pm SEM $(n=40 ; 25 \mathrm{M}, 15 \mathrm{~F})$ and were compared using the MIXED models procedure (SAS version 9.4; SAS Institute Inc., Cary, NC, USA) to test the effects of treatment, period, and treatment by period interactions. Baseline values were included as covariates for each endpoint. * Period 2 values > Period 1 values. Abbreviations: DBP, diastolic blood pressure; PP, pulse pressure; SBP, systolic blood pressure.

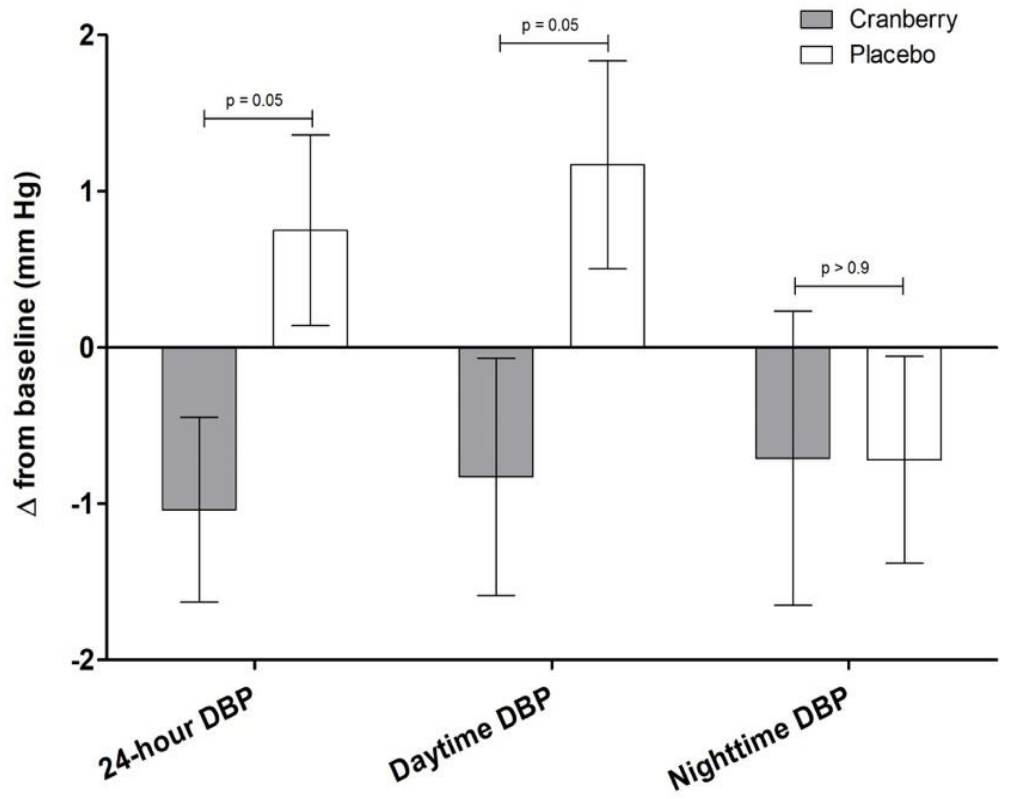

Figure 2. Change in ambulatory diastolic blood pressure following supplementation $(n=40)$. Change scores were calculated by subtracting study-entry baseline values from each post-supplementation measure. Values are means \pm SEM $(n=40 ; 25 \mathrm{M}, 15 \mathrm{~F})$ and were compared using the MIXED models procedure (SAS version 9.4; SAS Institute Inc., Cary, NC, USA) to test the effects of treatment, period, and treatment by period interactions. Baseline values were included as covariates for each endpoint. $p$-values represent significant main effects of treatment.

\subsection{Effects of Cranberry Juice on Atherogenic Lipoproteins and Other Blood Markers of CVD Risk}

There were no significant main effects of treatment on glucose/insulin, lipids, or markers of oxidative stress (Table 3 ). There was a significant treatment effect for large LDL particles $(p=0.02)$ and LDL size $(p=0.001)$ (Table 4 and Figure 3). Relative to baseline, cranberry juice increased large LDL particles by $8.7 \%$ compared to the $2 \%$ decrease following the placebo and increased LDL size by $0.3 \%$ compared to the $0.3 \%$ decrease following the placebo. There was no significant main effect of treatment for any measures 
of HDL efflux (Table 5 and Figure 4), but all significantly increased from baseline following both cranberry juice and placebo supplementation $(p \leq 0.02)$.

Table 3. Blood markers of cardiovascular disease risk in study participants $(n=40)$ following each 8 -week supplementation period ${ }^{1}$.

\begin{tabular}{|c|c|c|c|c|c|}
\hline & Baseline & Cranberry & Placebo & $\begin{array}{c}\text { Treatment } \\
p \text {-Value }\end{array}$ & $\begin{array}{c}\text { Period } \\
p \text {-Value }\end{array}$ \\
\hline Glucose $(\mathrm{mg} / \mathrm{dL}) \dagger$ & $92 \pm 1$ & $92 \pm 1$ & $94 \pm 1 *$ & 0.06 & 0.04 \\
\hline Insulin & $6.4 \pm 0.6$ & $6.6 \pm 0.4$ & $6.5 \pm 0.3$ & 0.9 & 0.8 \\
\hline Total Cholesterol (mg/dL) & $193 \pm 6$ & $192 \pm 3$ & $192 \pm 3$ & 0.8 & 0.8 \\
\hline HDL-C (mg/dL) & $48 \pm 2$ & $48 \pm 1$ & $48 \pm 1$ & 0.8 & 0.6 \\
\hline TC:HDL-C (mg/dL) & $4.2 \pm 0.2$ & $4.2 \pm 0.1$ & $4.2 \pm 0.1$ & $>0.9$ & 0.4 \\
\hline Direct LDL (mg/dL) & $124 \pm 5$ & $124 \pm 2$ & $121 \pm 3$ & 0.3 & 0.7 \\
\hline Non-HDL-C (mg/dL) & $144 \pm 6$ & $144 \pm 2$ & $144 \pm 3$ & 0.8 & 0.6 \\
\hline Triglycerides (mg/dL) & $110 \pm 7$ & $109 \pm 3$ & $115 \pm 6$ & 0.3 & 0.4 \\
\hline CRP (mg/L) & $2.3 \pm 0.4$ & $2.0 \pm 0.3$ & $1.7 \pm 0.2$ & 0.3 & 0.5 \\
\hline Isoprostanes $(\mathrm{ng} / \mathrm{mL})$ & $0.03 \pm 0.001$ & $0.03 \pm 0.002$ & $0.023 \pm 0.002$ & 0.7 & 0.5 \\
\hline
\end{tabular}

${ }^{1}$ All values are means $\pm \operatorname{SEM}(n=40 ; 25 \mathrm{M}, 15 \mathrm{~F})$ and were compared using the MIXED models procedure (SAS version 9.4; SAS Institute Inc., Cary, NC, USA) to test the effects of treatment, period, and treatment by period interactions. Baseline values were included as covariates for each endpoint. * indicates a significant change from baseline $(p=0.05)$. + Period 2 values $>$ Period 1 values. Abbreviations: CRP, C-reactive protein; HDL-C, high-density lipoprotein-cholesterol; LDL-C, low-density lipoprotein-cholesterol; TC, total cholesterol.

Table 4. Lipoprotein particle number, particle size, and other lipid profile characteristics in study participants $(n=40)$ following each 8-week supplementation period ${ }^{1}$.

\begin{tabular}{|c|c|c|c|c|c|c|}
\hline & & Baseline & Cranberry & Placebo & $\begin{array}{l}\text { Treatment } \\
p \text {-Value }\end{array}$ & $\begin{array}{c}\text { Period } \\
p \text {-Value }\end{array}$ \\
\hline \multirow{4}{*}{$\begin{array}{l}\text { VLDL and Chylomicron } \\
\text { Particle Concentrations }\end{array}$} & $\begin{array}{l}\text { Total VLDL and Chylomicron } \\
\text { Particles (nmol/L) }\end{array}$ & $44.71 \pm 1.72$ & $47.40 \pm 2.57$ & $50.19 \pm 2.29 *$ & 0.3 & 0.9 \\
\hline & $\begin{array}{l}\text { Large VLDL and Chylomicron } \\
\text { Particles (nmol/L) }\end{array}$ & $4.42 \pm 0.39$ & $4.59 \pm 0.35$ & $4.66 \pm 0.44$ & 0.9 & 0.2 \\
\hline & $\begin{array}{l}\text { Medium VLDL Particles } \\
(\mathrm{nmol} / \mathrm{L})\end{array}$ & $17.63 \pm 1.35$ & $17.23 \pm 1.48$ & $20.61 \pm 1.87$ & 0.06 & $>0.9$ \\
\hline & $\begin{array}{l}\text { Small VLDL Particles } \\
(\mathrm{nmol} / \mathrm{L})\end{array}$ & $22.68 \pm 1.09$ & $25.59 \pm 2.00$ & $24.94 \pm 1.47$ & 0.7 & 0.7 \\
\hline \multirow{4}{*}{$\begin{array}{l}\text { LDL Particle } \\
\text { Concentrations }\end{array}$} & Total LDL Particles (nmol/L) & $1210.15 \pm 38.92$ & $1180.38 \pm 22.80$ & $1185.35 \pm 30.59$ & 0.9 & 0.3 \\
\hline & IDL Particles (nmol/L) & $245.3 \pm 16.73$ & $226.43 \pm 16.90$ & $228.65 \pm 17.81$ & 0.9 & 0.5 \\
\hline & Large LDL Particles (nmol/L) & $339 \pm 18.00$ & $368.53 \pm 16.63$ & $332.30 \pm 17.61$ & 0.02 & 0.2 \\
\hline & Small LDL Particles (nmol/L) & $625.83 \pm 20.13$ & $585.30 \pm 20.23 *$ & $624.33 \pm 20.51$ & 0.09 & 0.8 \\
\hline \multirow{4}{*}{$\begin{array}{l}\text { HDL Particle } \\
\text { Concentrations }\end{array}$} & Total HDL Particles $(\mu \mathrm{mol} / \mathrm{L})$ & $31.51 \pm 0.52$ & $30.87 \pm 0.61$ & $31.55 \pm 0.52$ & 0.2 & 0.1 \\
\hline & Large HDL Particles $(\mu \mathrm{mol} / \mathrm{L})$ & $6.12 \pm 0.32$ & $5.96 \pm 0.24$ & $6.00 \pm 0.23$ & 0.8 & 0.9 \\
\hline & $\begin{array}{l}\text { Medium HDL Particles } \\
(\mu \mathrm{mol} / \mathrm{L})\end{array}$ & $9.17 \pm 0.76$ & $8.84 \pm 0.49$ & $8.65 \pm 0.69$ & 0.8 & 0.7 \\
\hline & Small HDL Particles $(\mu \mathrm{mol} / \mathrm{L})$ & $16.21 \pm 0.70$ & $16.13 \pm 0.58$ & $16.84 \pm 0.75$ & 0.2 & $0.03+$ \\
\hline \multirow{3}{*}{ Mean Particle Sizes } & VLDL Size (nm) & $50.26 \pm 0.72$ & $49.84 \pm 0.78$ & $49.31 \pm 0.89$ & 0.6 & 0.3 \\
\hline & LDL Size (nm) & $20.79 \pm 0.06$ & $20.86 \pm 0.04$ & $20.72 \pm 0.05$ & 0.001 & 0.7 \\
\hline & HDL Size (nm) & $9.15 \pm 0.05$ & $9.16 \pm 0.04$ & $9.12 \pm 0.04$ & 0.3 & 0.4 \\
\hline
\end{tabular}


Table 4. Cont.

\begin{tabular}{cccccc}
\hline & Baseline & Cranberry & Placebo & $\begin{array}{c}\text { Treatment } \\
p \text {-Value }\end{array}$ & $\begin{array}{c}\text { Period } \\
p \text {-Value }\end{array}$ \\
\hline & Total Triglyceride (mg/dL) & $114.6 \pm 5.38$ & $115.97 \pm 5.13$ & $121.15 \pm 6.58$ & 0.4 \\
\cline { 2 - 6 } Calculated Lipids & $\begin{array}{c}\text { Total VLDL and Chylomicron } \\
\text { Triglyceride (mg/dL) }\end{array}$ & $76.7 \pm 3.85$ & $78.88 \pm 3.79$ & $83.28 \pm 4.57$ & 0.4 \\
\cline { 2 - 6 } & $\begin{array}{c}\text { Total HDL Cholesterol } \\
\text { (mg/dL) }\end{array}$ & $48.55 \pm 1.52$ & $47.83 \pm 1.10$ & $47.78 \pm 1.1$ & $>0.9$ \\
\hline $\begin{array}{c}\text { Lipoprotein } \\
\text { Insulin-Resistance Score } \\
\text { (LPIR) }\end{array}$ & $50.6 \pm 2.28$ & $49.03 \pm 1.80$ & $50.10 \pm 2.08$ & 0.3 \\
\hline
\end{tabular}

${ }^{1}$ All values are means \pm SEM $(n=40 ; 25 \mathrm{M}, 15 \mathrm{~F})$ and were compared using the MIXED models procedure (SAS version 9.4; SAS Institute Inc., Cary, NC, USA) to test the effects of treatment, period, and treatment by period interactions. Baseline values were included as covariates for each endpoint. * indicates a significant change from baseline $(p \leq 0.05)$. + Period 1 values $>$ Period 2 values. Abbreviations: HDL, high-density lipoprotein; LDL, low-density lipoprotein; VLDL, very low-density lipoprotein, VLDL.
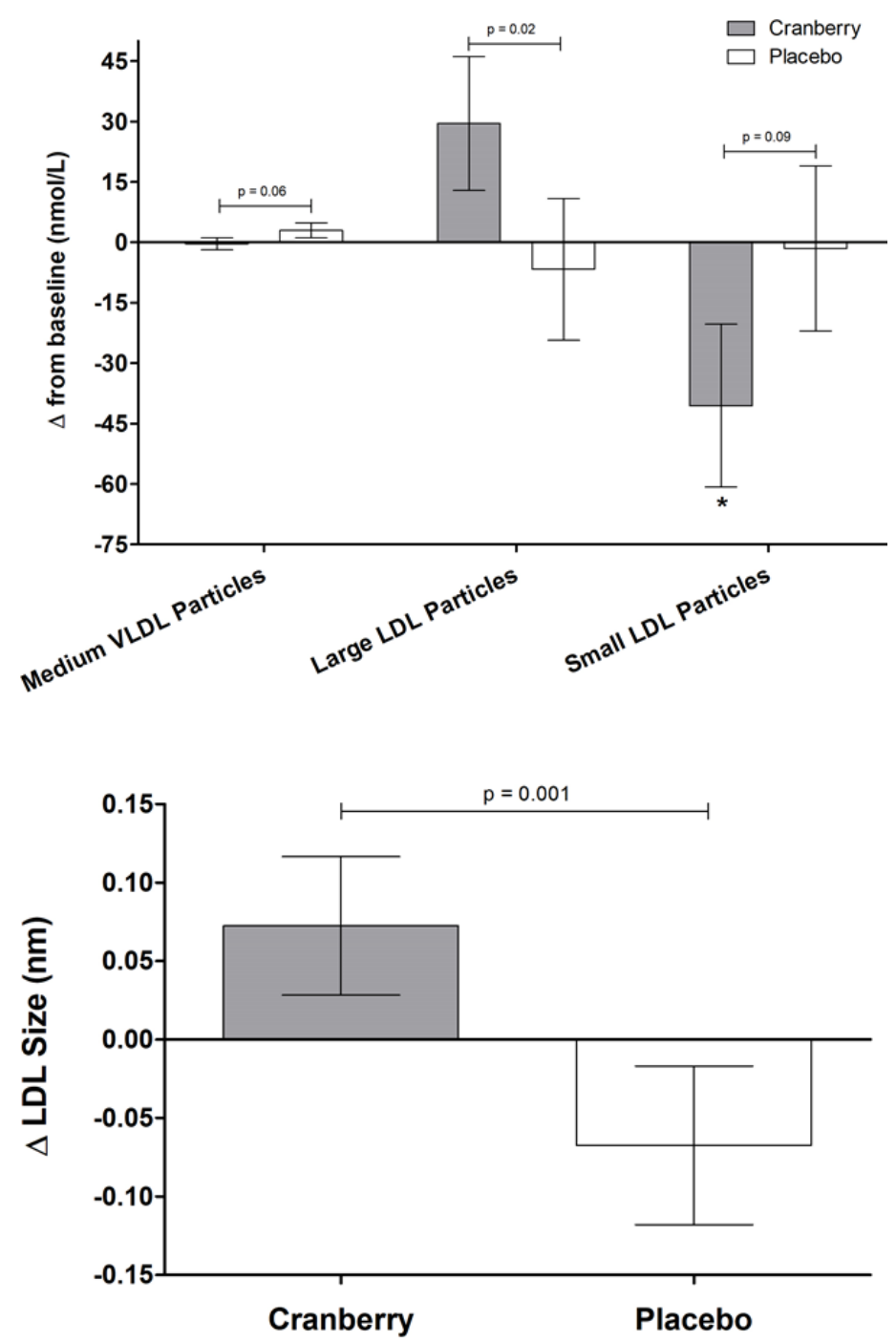

Figure 3. Changes in lipoprotein sub-fractions and characteristics following supplementation. Change scores were calculated by subtracting study-entry baseline values from each postsupplementation measure. Values are means $\pm \operatorname{SEM}(n=40 ; 25 \mathrm{M}, 15 \mathrm{~F})$ and were compared using the MIXED models procedure (SAS version 9.4; SAS Institute Inc., Cary, NC, USA) to test the effects of treatment, period, and treatment by period interactions. Baseline values were included as covariates for each endpoint. $p$-values represent treatment effects, and * indicates a significant change from baseline $(p=0.05)$. 
Table 5. Ex vivo cholesterol efflux (\% efflux per $4 \mathrm{~h}$ ) in study participants $(n=40)$ following each 8-week supplementation period ${ }^{1}$.

\begin{tabular}{cccccc}
\hline & Baseline & Cranberry & Placebo & $\begin{array}{c}\text { Treatment } \\
p \text {-Value }\end{array}$ & $\begin{array}{c}\text { Period } \\
p \text {-Value }\end{array}$ \\
\hline ABCA1 & $2.29 \pm 0.12$ & $2.86 \pm 0.16^{*}$ & $2.91 \pm 0.15^{*}$ & 0.8 & 0.09 \\
\hline Global efflux (+cAMP) & $7.19 \pm 0.20$ & $8.16 \pm 0.23^{*}$ & $8.29 \pm 0.25^{*}$ & 0.7 & 0.8 \\
\hline ABCA1-independent (-cAMP) & $4.89 \pm 0.11$ & $5.30 \pm 0.17^{*}$ & $5.38 \pm 0.14^{*}$ & 0.7 & 0.2 \\
\hline
\end{tabular}

${ }^{1}$ All values are means \pm SEM $(n=40 ; 25 \mathrm{M}, 15 \mathrm{~F})$ and were compared using the MIXED models procedure (SAS version 9.4; SAS Institute Inc., Cary, NC, USA) to test the effects of treatment, period, and treatment by period interactions. Baseline values were included as covariates for each endpoint. ABCA1-specific efflux was calculated as the difference between cAMP-stimulated and non-stimulated (-cAMP) efflux. * indicates a significant change from baseline $(p \leq 0.02)$. Abbreviations: ABCA1, ATP-binding cassette transporter 1; cAMP, cyclic adenosine monophosphate.

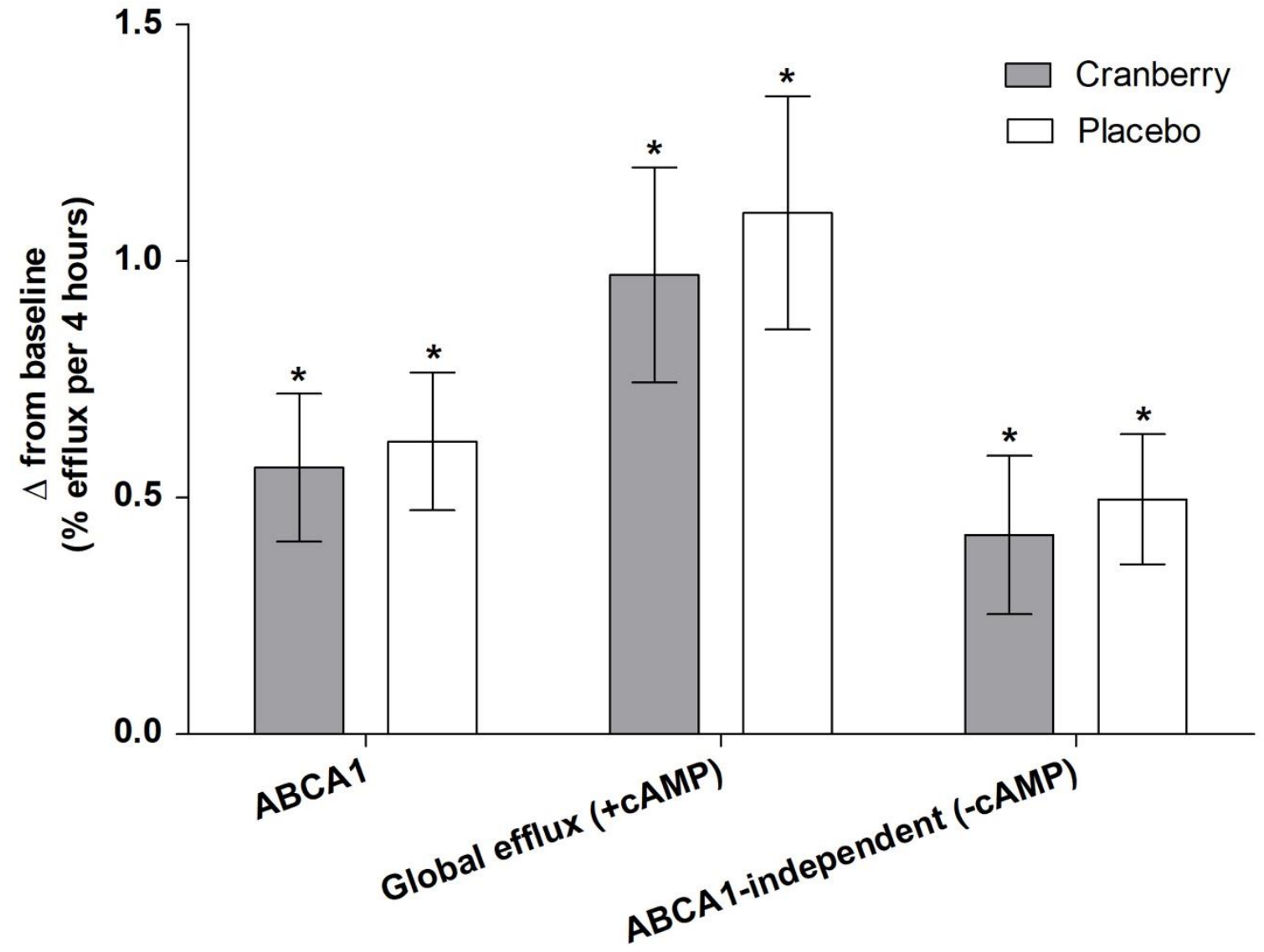

Figure 4. Change in ex vivo measures of HDL-C efflux following supplementation. Change scores were calculated by subtracting study-entry baseline values from each post-supplementation measure. Values are means \pm SEM $(n=40 ; 25 \mathrm{M}$, $15 \mathrm{~F}$ ) and were compared using the MIXED models procedure (SAS version 9.4; SAS Institute Inc., Cary, NC, USA) to test the effects of treatment, period, and treatment by period interactions. Baseline values were included as covariates for each endpoint. * indicates a significant change from baseline $(p<0.05)$.

\subsection{Effects of Cranberry Juice on HDL Efflux and Role of BMI}

Exploratory subgroup analyses according to BMI category identified a significant interaction between treatment and BMI for global HDL efflux (+cAMP) (interaction $p$ value $=0.02$; Figure 5). Post hoc comparisons demonstrated that cranberry juice supplementation was more effective in participants with overweight compared to both individuals of normal weight and individuals with obesity ( $p=0.009$ and $p=0.04$, respectively). Cranberry juice also significantly increased global HDL efflux (+cAMP) compared to the placebo in individuals with overweight $(p=0.05)$, whereas there was a greater increase with the 
placebo in participants of normal body weight $(p=0.02)$. There was no significant difference between the effect of cranberry juice and placebo in individuals with obesity. There was not a significant interaction between treatment and BMI category for other measures of HDL efflux (data not shown; $p>0.05$ ).

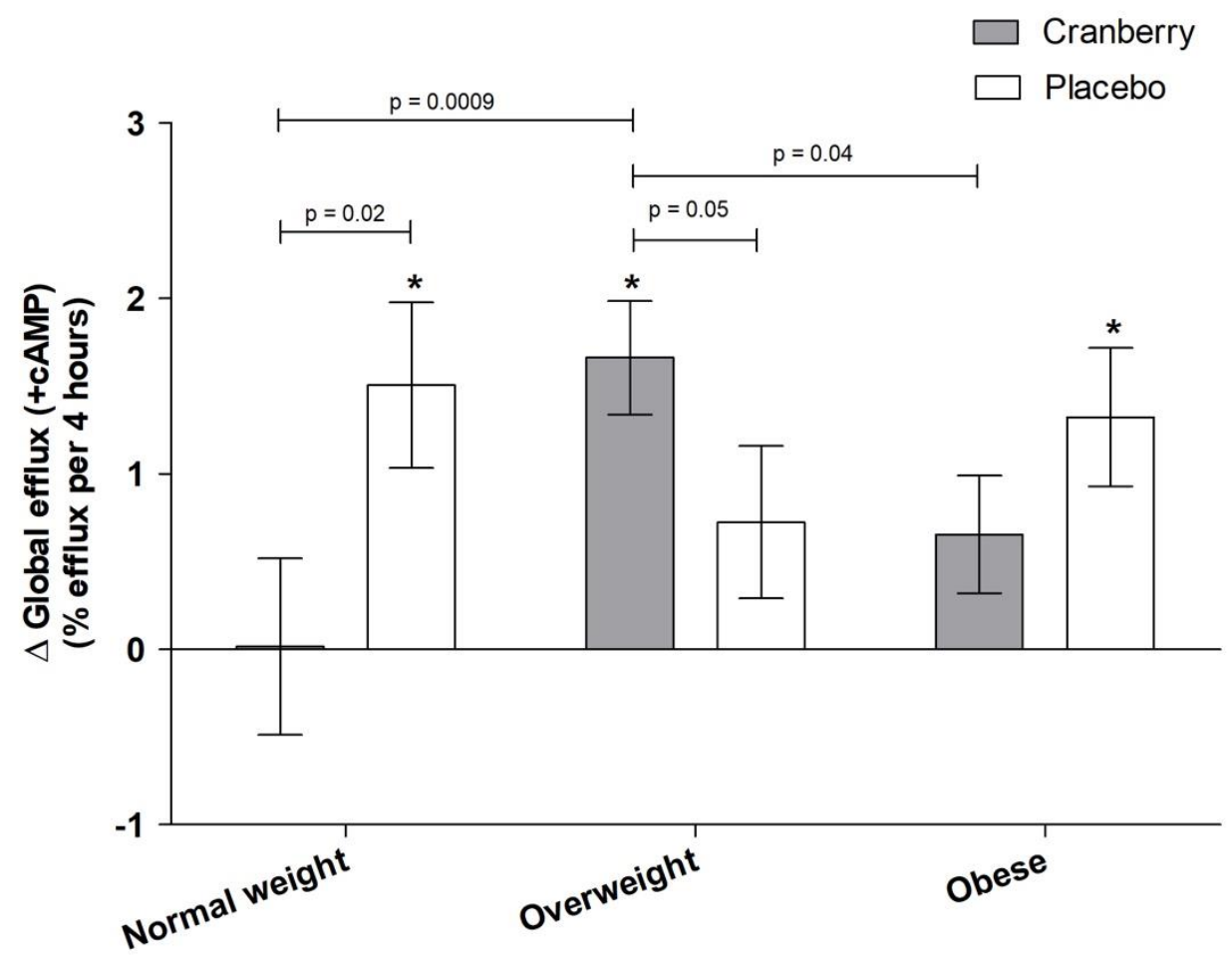

Figure 5. Change in global HDL efflux (+cAMP) following supplementation according to BMI category. Change scores were calculated by subtracting study-entry baseline values from each postsupplementation measure. Values are means $\pm \operatorname{SEM}(n=40 ; 25 \mathrm{M}, 15 \mathrm{~F})$ and were compared using the MIXED models procedure (SAS version 9.4; SAS Institute Inc., Cary, NC, USA) to test the effects of treatment, period, and treatment by period interactions. Baseline values were included as covariates for each endpoint. $p$-values represent unadjusted post hoc comparisons, and * indicates a significant change from baseline $(p \leq 0.05)$.

\subsection{Baseline CRP as a Moderator of Treatment Effects}

Exploratory secondary analyses revealed significant differences in the effect of supplementation depending on baseline CRP status (defined as high or low compared to the median baseline CRP value of $1.3 \mathrm{mg} / \mathrm{L}$ ). Baseline characteristics for participants according to CRP status are provided in Table S3. With respect to measures of vascular function, there was a significant treatment by CRP interaction for brachial systolic blood pressure $(p=0.001)$, central systolic blood pressure $(p=0.03)$, and central pulse pressure $(p=0.009)$ (Figure 6). In post hoc analyses, there was a clear distinction in the effect of cranberry supplementation depending on CRP status, with a $4.8 \%$ decrease in brachial SBP, $4 \%$ decrease in central SBP, and $12 \%$ decrease in central PP in individuals with low CRP compared to individuals with high CRP.

There was also a significant treatment by CRP interaction for total cholesterol $(p=0.05)$, HDL-C $(p=0.03)$, non-HDL-C $(p=0.04)$, and glucose $(p=0.04)$, with post hoc analyses indicating clear differences in the response to treatment in individuals with low CRP compared to high CRP (data not shown). Changes in lipoprotein sub-fractions were also influenced by baseline CRP status (data not shown). There was a significant interaction for calculated HDL-C $(p=0.007)$, HDL particles $(p=0.01)$, and the lipoprotein insulin-resistance score (LPIR; $p=0.04$ ), a composite metabolic score that incorporates the multi-faceted role of insulin resistance on lipoprotein metabolism [32,33]. In post hoc analyses, calculated 
HDL-C remained stable in all participants following cranberry supplementation, whereas individuals with high CRP experienced a $4 \mathrm{mg} / \mathrm{dL}$ decrease in HDL-C following the placebo compared to individuals with low CRP. In terms of HDL particles, there was a significant decrease following both cranberry and placebo supplementation in individuals with high CRP. For LPIR, cranberry supplementation lowered LPIR by $17 \%$ in individuals with low CRP compared to participants with a high baseline CRP.
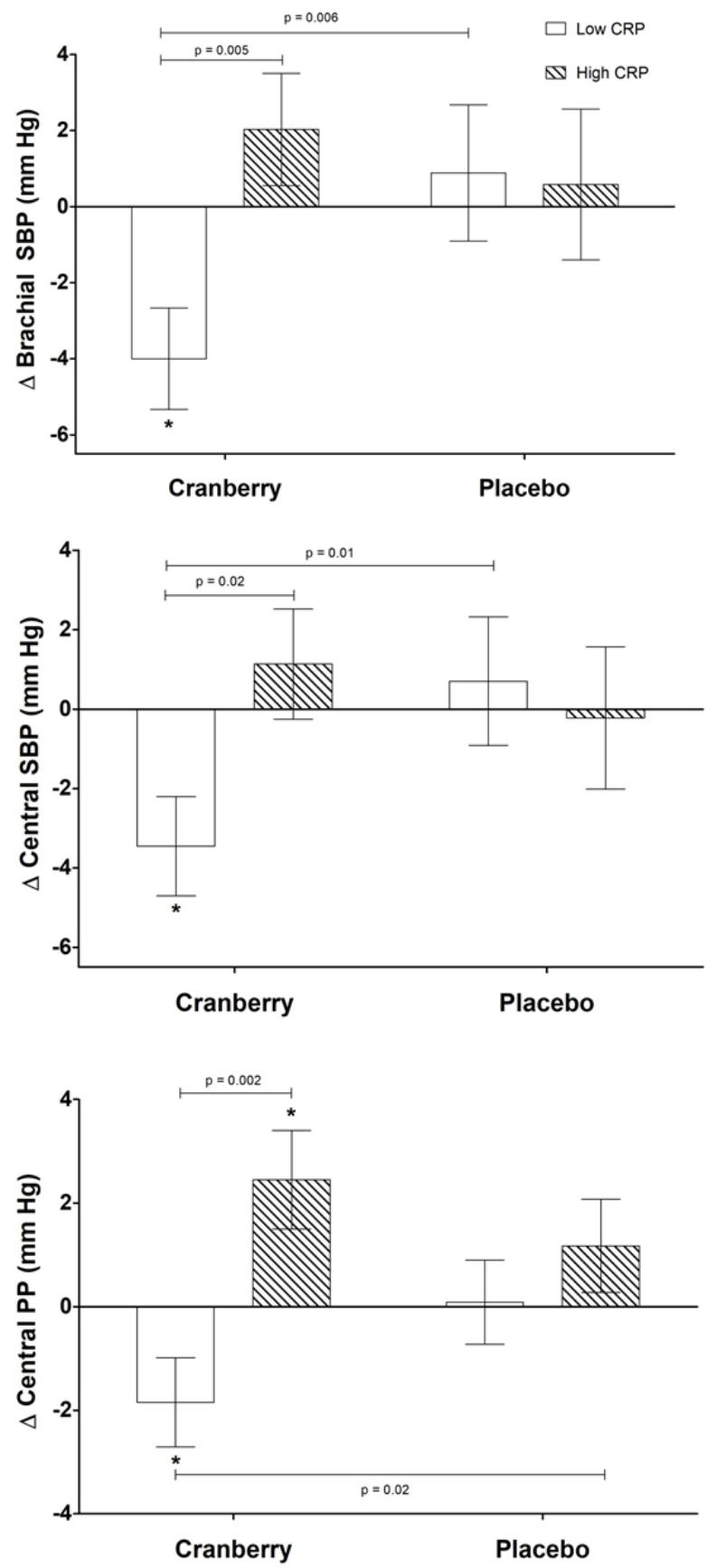

Figure 6. Significant changes in vascular function according to baseline CRP status. Change scores were calculated by subtracting study-entry baseline values from each post-supplementation measure. Values are means \pm SEM $(n=40 ; 25 \mathrm{M}, 15 \mathrm{~F})$ and were compared using the MIXED models procedure (SAS version 9.4; SAS Institute Inc., Cary, NC, USA). Baseline values were included as covariates for each endpoint. CRP status was defined based on the median baseline CRP value of $1.3 \mathrm{mg} / \mathrm{L}$. $p$-values indicate unadjusted post hoc comparisons, and * indicates a significant change from baseline $(p \leq 0.05)$. 


\section{Discussion}

This study was designed to evaluate the effect of cranberry juice on CVD risk factors in adults with elevated blood pressure. There was no effect of cranberry juice supplementation on the primary endpoint of central systolic blood pressure or for central or brachial diastolic blood pressure. Compared to the placebo, diastolic ambulatory blood pressure was lower following cranberry juice supplementation; the between-treatment difference occurred during daytime hours rather than during nighttime. No other vascular effects were observed. Cranberry juice significantly increased the concentration of large LDL particles and LDL size compared to the placebo. This coincided with a significant decrease from baseline in the concentration of small LDL particles following cranberry juice, but this was not significant compared to the control (treatment $p$-value $=0.09$ ). There was no main effect of treatment on ex vivo HDL efflux, but exploratory subgroup analyses indicate that cranberry juice may be more effective for increasing global HDL efflux (+cAMP) in individuals with overweight compared to those who are normal weight or classified as obese. Our exploratory analyses investigating the effect of baseline CRP also indicate that inflammatory status may potentially alter the effect of cranberry juice supplementation and that there may be greater benefits for vascular function and atherogenic lipoproteins in individuals with lower levels of inflammation.

Previous studies of cranberry supplementation have found mixed results for vascular function endpoints. Epidemiological, in vitro, and animal studies all support the hypothesis that cranberries may be beneficial for vascular function [34,35] - potentially via mechanisms related to their bioactive compounds $[5,8]$. A recent meta-analysis of cranberry supplementation studies found a significant reduction in brachial systolic blood pressure $(-3.6 \mathrm{~mm} \mathrm{Hg} ; 95 \% \mathrm{CI}:-6.27,-0.98)$, an effect that was more apparent in participants who were 50 years or older [8]. Ambulatory blood pressure has been shown to improve CVD risk stratification beyond traditional risk factors, including conventional blood pressure measurement [36-38], and previous studies of polyphenol-rich dietary supplements have found significant improvements for both systolic and diastolic ambulatory blood pressure $[39,40]$. However, null results have been found in other studies $[41,42]$. To our knowledge, this is the first reported effect for cranberry juice supplementation on ambulatory diastolic blood pressure (as opposed to seated blood pressure). Additional research is needed to verify this effect, but it could present a promising means of CVD risk reduction with cranberry juice consumption.

In terms of central blood pressure and indices of arterial stiffness, clinical studies of antioxidant-rich foods, such as grapefruit juice [43], tart cherry juice [44], blueberries [45,46], pomegranate juice [47,48], and purple potatoes [49], have found significant benefits. Few cranberry studies have reported effects on these endpoints. No effect on AI was found in overweight men with a low-calorie cranberry juice [11], but four weeks of supplementation with double-strength cranberry juice significantly reduced PWV in participants with stable coronary artery disease [12]. Comparatively, our study participants did not have CVD, and the moderately elevated brachial blood pressure of our participants at baseline (mean systolic and diastolic blood pressure $=124 / 81 \mathrm{~mm} \mathrm{Hg}$ ) may not have been high enough to observe a significant effect of the cranberry treatment [50]. The absence of consistent changes in vascular function and arterial stiffness may also be due to the specific bioactive profile provided by each supplement [39] and/or be related to the plasma bioavailability of cranberry bioactives. Pharmacokinetic studies indicate that plasma availability of cranberry bioactives is very low ( $<1 \%$ of consumed quantities), with high inter-individual variability. Peak plasma concentrations occur over 1-8 h [51-53], although there remains debate about whether this occurs earlier $(\sim 1-3 \mathrm{~h})$ [53] or later $(\sim 6-10 \mathrm{~h})[51,52]$ in that time period. Regardless, this would suggest that the concentration of cranberry bioactives in plasma is very low or non-existent following a 12-h fast and that effects may be more apparent acutely [54]. Furthermore, despite the short half-life of phenolic compounds in plasma, the concentrations are sufficient to induce changes in gene expression and signal transduction $[51,53]$. Studies have also found that cranberry polyphenol metabolites are 
abundant in plasma, and these compounds may be the more physiologically active form, although their correlation with vascular function is unclear [52]. There is also evidence of tissue accumulation of anthocyanins in animals following long-term feeding [55], suggesting that repeated exposure over time may confer benefits beyond the acute state. No acute effect of cranberry supplementation has been shown for PWV or AI [52,56], but very few studies have been conducted and in very limited participant populations.

Dietary changes have been shown to be effective for improving the lipid/lipoprotein profile, including LDL-C and HDL-C, but evidence for cranberries remains inconsistent. For instance, a recent meta-analysis of berry-supplementation studies (including nine cranberry studies) found a significant reduction in LDL-C but no change in HDL-C, total cholesterol, triglycerides, apolipoprotein B, or apolipoprotein A-I [57]. Conversely, a metaanalysis focused exclusively on cranberry studies $(n=10)$ found a significant increase in HDL-C, particularly for participants younger than 50 years of age, but no effect on total cholesterol, LDL-C, or triglycerides [8]. With regard to individual studies of cranberry juice, supplementation significantly increased HDL-C in adults with abdominal obesity [13], reduced triglycerides in healthy adults [14], and reduced apolipoprotein $B$ and increased apolipoprotein A-I in patients with type 2 diabetes [15] but had no effect in other studies [16-19]. Comparatively, we found no effect when using a similar dose of cranberry juice and supplementation duration. It is possible that the baseline lipid profile (mean LDL-C $=124 \pm 5 \mathrm{mg} / \mathrm{dL}$ ) of our study population provided less opportunity to observe significant effects.

Lipoprotein subfractions, defined according to particle size and density, may provide additional insight into CVD risk but do not serve as a replacement for the standard lipid risk factors [58-61]. With regard to cranberries, supplementation with cranberry juice was found to increase apolipoprotein A-I and decrease apolipoprotein B in patients with type 2 diabetes [15]. We found that cranberry juice significantly increased the concentration of large LDL particles and decreased overall LDL size. This occurred in conjunction with a decrease in small LDL particles compared to baseline, although the main effect of treatment compared to the control did not reach statistical significance $(p=0.09)$. However, given the evidence that LDL size may be a significant factor in assessing CVD risk [58,59], further research into the effects of cranberries on lipoprotein subfractions is warranted.

HDL cholesterol efflux capacity, a measure of reverse cholesterol transport, has been shown to be a predictor of CVD risk and atherosclerosis $[20,62,63]$. There is some evidence for beneficial effects of cranberries on HDL-C [64,65], but effects on ex vivo HDL efflux have not been reported. Other bioactive-rich supplements have shown mixed results for ex vivo HDL efflux, with significant improvements following anthocyanin supplementation (160 mg/d for 24 weeks) [66] but null results for foods/supplements, such as soy protein [22]. We found no main effect of treatment on either global or transporter-specific cholesterol efflux. However, when participants were stratified by BMI (normal weight, overweight, and obese) in exploratory analyses, there was a significant treatment interaction, with cranberry juice having a greater effect on global efflux in overweight participants compared to those who had obesity or were of normal weight. This could be due to the relative metabolic health of these individuals, with there being no room for improvement in healthy-weight individuals and more severe dysregulation in individuals with obesity that would require more potent interventions. However, this is in contrast to previous studies that have shown more beneficial effects in normal-weight participants [67]. It is also unclear why the placebo juice had a beneficial effect on global cholesterol efflux in the normal-weight participants in the current study compared to cranberry juice. However, this suggests that the baseline health status of participants may play a determining role in the potential effects of cranberry juice supplementation and warrants further investigation.

Similarly, our exploratory analyses suggest that baseline inflammatory status may also determine the effects of cranberry juice supplementation on specified CVD risk factors. Elevated CRP (in the absence of any identifiable acute cause, such as infection, illness, physical trauma, etc.) is indicative of underlying systemic inflammation and higher CVD 
risk [68]. Thus, individuals with elevated CRP may be experiencing a greater physiological challenge that cannot be overcome by cranberry juice bioactives alone. To our knowledge, no previous studies have investigated a potential interaction with baseline CRP or other inflammatory markers. Additional research is needed to investigate and confirm this potential influence of baseline inflammatory status on the effect of cranberry juice supplementation.

\section{Strengths and Limitations}

The crossover design of this study allowed participants to act as their own controls and minimize the influence of between-subject variability. It should be noted that $p$ values for secondary analyses and post hoc analyses were not corrected for multiple comparisons, which increases the risk of type 1 error. Our sample size was relatively large $(n=40)$, and our participants were recruited specifically on the basis of resting brachial blood pressure to maximize the potential for observing treatment effects relevant to blood pressure management. According to the American Heart Association's blood pressure categories, the mean brachial blood pressure of our study population (mean $=124 / 81 \mathrm{~mm}$ $\mathrm{Hg}$ ) would be categorized as hypertensive (i.e., brachial blood pressure $80-89 \mathrm{~mm} \mathrm{Hg}$ ) [69]; however, this degree of blood pressure elevation might have been insufficient to see an effect of the cranberry juice supplement. This and other baseline health characteristics may have reduced the potential for cranberry supplementation to exert significant changes. The cranberry intervention was incorporated into the normal dietary habits of participants, making it more reflective of real world implementation. However, no data were collected on the background diet of participants, and there was no formal oversight to ensure that participants followed the instructions to maintain their normal baseline diet for the duration of the study. This prevents us from conclusively determining whether other diet/lifestyle changes may have occurred and influenced study outcomes. The dose of cranberry juice was consistent with previous studies and represents an easily achievable daily amount. The cranberry and placebo juices were also closely matched for flavor and appearance to maintain blinding and for macronutrients in order to isolate the effect of cranberry bioactives. However, although cranberry juice remains a key source of proanthocyanidins and other bioactive compounds, it should be noted that the multi-step processing methods can lead to a substantial loss of these compounds, particularly anthocyanins, which are more vulnerable to degradation $[5,7,70]$. Therefore, the type of cranberry supplement (fresh, juice, extract, etc.) may have important implications for clinical effects.

\section{Conclusions}

Supplementation with cranberry juice for eight weeks did not significantly alter the primary endpoint of central systolic blood pressure in adults with overweight and elevated blood pressure; however, the concentration of large LDL particles and LDL size significantly increased without affecting LDL-C concentration. Cranberry juice also significantly lowered 24-h diastolic ambulatory blood pressure. No beneficial effects of cranberry juice were found for central or brachial diastolic pressure or other measures of vascular function. Exploratory subgroup analyses indicated that BMI and CRP may influence effects, and the potential role of baseline health status should be investigated further in future studies. Additional research on other emerging CVD risk factors, such as the gut microbiome, would help to clarify the potential of cranberries for reducing CVD risk.

Supplementary Materials: The following are available online at https:/ / www.mdpi.com/article/10 $.3390 /$ nu13082618/s1, Table S1: Nutrient profile of the cranberry and placebo beverages, Table S2: Phenolic content of cranberry and placebo beverages (mg per $8 \mathrm{fl} \mathrm{oz}$ ), Table S3: Baseline characteristics of study participants by CRP status, Figure S1: Significant treatment by sex interactions following supplementation.

Author Contributions: The authors' responsibilities were as follows-C.K.R., A.C.S.-R. and P.M.K.-E. designed the research; S.M. conducted the research; C.K.R., A.C.S.-R. and T.L.G. performed the 
statistical analyses; and C.K.R., A.C.S.-R., K.S.P. and P.M.K.-E. wrote the manuscript. All authors take responsibility for the manuscript's final content. All authors have read and agreed to the published version of the manuscript.

Funding: This research was funded by Ocean Spray and the Penn State Clinical \& Translational Research Institute, Pennsylvania State University CTSA, NIH/NCATS Grant Number UL1 TR000127.

Institutional Review Board Statement: This study was conducted according to the guidelines issued in the Declaration of Helsinki, and all procedures involving human subjects were approved by the Institutional Review Board of the Pennsylvania State University. Written informed consent was obtained from all participants. The study is registered at clinicaltrials.gov as NCT02556749.

Informed Consent Statement: Informed consent was obtained from all subjects involved in the study.

Data Availability Statement: The data presented in this study are available on request from the corresponding author.

Acknowledgments: We express our gratitude to our research participants for their dedication to the project as well as the nursing and clinical staff of the Clinical Research Center of the Pennsylvania State University. We would also like to thank Danette Teeter for her dedicated work on this study and crucial role in participant recruitment and data collection. In addition, we appreciate Caitlin Lynch's help with recruitment and sample collection. Ocean Spray provided financial support for the study. This project was also supported by the Penn State Clinical \& Translational Research Institute, Pennsylvania State University CTSA, NIH/NCATS Grant Number UL1 TR000127. The contents are solely the responsibility of the authors and do not necessarily represent the official views of the NIH or NCATS. Financial supporters had no role in the design and conduct of the study; in the collection, analysis, and interpretation of the data; or in the preparation, review, or approval of the manuscript.

Conflicts of Interest: The authors declare no conflict of interest.

\section{References}

1. Centers for Disease Control. National Vital Statistics Report_Deaths: Leading causes for 2017; U.S. Department of Health \& Human Services: Hyattsville, MD, USA, 2019.

2. World Health Organization. The Top 10 Causes of Death. 2018. Available online: https://www.who.int/news-room/fact-sheets/ detail/the-top-10-causes-of-death (accessed on 29 July 2021).

3. 2015 Dietary Guidelines Advisory Committee. Scientific Report of the 2015 Dietary Guidelines Advisory Committee; U.S. Department of Agriculture: Washington, DC, USA, 2015.

4. Eckel, R.H.; Jakicic, J.M.; Ard, J.D.; de Jesus, J.M.; Houston Miller, N.; Hubbard, V.S.; Lee, I.; Lichtenstein, A.H.; Loria, C.M.; Millen, B.E.; et al. 2013 AHA/ACC guideline on lifestyle management to reduce cardiovascular risk: A report of the American College of Cardiology/American Heart Association Task Force on Practice Guidelines. J. Am. Coll. Cardiol. 2014, 63, 2960-2984. [CrossRef]

5. Blumberg, J.B.; Camesano, T.A.; Cassidy, A.; Kris-Etherton, P.; Howell, A.; Manach, C.; Ostertag, L.M.; Sies, H.; Skulas-Ray, A.; Vita, J.A. Cranberries and their bioactive constituents in human health. Adv. Nutr. 2013, 4, 618-632. [CrossRef] [PubMed]

6. Neto, C.C. Cranberry and blueberry: Evidence for protective effects against cancer and vascular diseases. Mol. Nutr. Food Res. 2007, 51, 652-664. [CrossRef] [PubMed]

7. Cote, J.; Caillet, S.; Doyon, G.; Sylvain, J.F.; Lacroix, M. Bioactive compounds in cranberries and their biological properties. Crit. Rev. Food Sci. Nutr. 2010, 50, 666-679. [CrossRef] [PubMed]

8. Pourmasoumi, M.; Hadi, A.; Najafgholizadeh, A.; Joukar, F.; Mansour-Ghanaei, F. The effects of cranberry on cardiovascular metabolic risk factors: A systematic review and meta-analysis. Clin. Nutr. 2019, 39, 774-788. [CrossRef]

9. Roman, M.J.; Devereux, R.B.; Kizer, J.R.; Lee, E.T.; Galloway, J.M.; Ali, T.; Umans, J.G.; Howard, B.V. Central pressure more strongly relates to vascular disease and outcome than does brachial pressure: The Strong Heart Study. Hypertension 2007, 50, 197-203. [CrossRef]

10. Waddell, T.K.; Dart, A.M.; Medley, T.L.; Cameron, J.D.; Kingwell, B.A. Carotid pressure is a better predictor of coronary artery disease severity than brachial pressure. Hypertension 2001, 38, 927-931. [CrossRef]

11. Ruel, G.; Lapointe, A.; Pomerleau, S.; Couture, P.; Lemieux, S.; Lamarche, B.; Couillard, C. Evidence that cranberry juice may improve augmentation index in overweight men. Nutr. Res. 2013, 33, 41-49. [CrossRef]

12. Dohadwala, M.M.; Holbrook, M.; Hamburg, N.M.; Shenouda, S.M.; Chung, W.B.; Titas, M.; Kluge, M.A.; Wang, N.; Palmisano, J.; Milbury, P.E.; et al. Effects of cranberry juice consumption on vascular function in patients with coronary artery disease. Am. J. Clin. Nutr. 2011, 93, 934-940. [CrossRef]

13. Chew, B.; Mathison, B.; Kimble, L.; McKay, D.; Kaspar, K.; Khoo, C.; Chen, C.O.; Blumberg, J. Chronic consumption of a low calorie, high polyphenol cranberry beverage attenuates inflammation and improves glucoregulation and HDL cholesterol in healthy overweight humans: A randomized controlled trial. Eur. J. Nutr. 2019, 58, 1223-1235. [CrossRef] 
14. Novotny, J.A.; Baer, D.J.; Khoo, C.; Gebauer, S.K.; Charron, C.S. Cranberry juice consumption lowers markers of cardiometabolic risk, including blood pressure and circulating C-reactive protein, triglyceride, and glucose concentrations in adults. J. Nutr. 2015, 145, 1185-1193. [CrossRef]

15. Shidfar, F.; Heydari, I.; Hajimiresmaiel, S.J.; Hosseini, S.; Shidfar, S.; Amiri, F. The effects of cranberry juice on serum glucose, apoB, apoA-I, Lp(a), and Paraoxonase-1 activity in type 2 diabetic male patients. J. Res. Med. Sci. 2012, 17, 355-360.

16. Basu, A.; Betts, N.M.; Ortiz, J.; Simmons, B.; Wu, M.; Lyons, T.J. Low-energy cranberry juice decreases lipid oxidation and increases plasma antioxidant capacity in women with metabolic syndrome. Nutr. Res. 2011, 31, 190-196. [CrossRef] [PubMed]

17. Duthie, S.J.; Jenkinson, A.M.; Crozier, A.; Mullen, W.; Pirie, L.; Kyle, J.; Yap, L.S.; Christen, P.; Duthie, G.G. The effects of cranberry juice consumption on antioxidant status and biomarkers relating to heart disease and cancer in healthy human volunteers. Eur. J. Nutr. 2006, 45, 113-122. [CrossRef] [PubMed]

18. Chambers, B.K.; Camire, M.E. Can cranberry supplementation benefit adults with type 2 diabetes? Diabetes Care 2003, 26, 2695-2696. [CrossRef]

19. Paquette, M.; Medina Larque, A.S.; Weisnagel, S.J.; Desjardins, Y.; Marois, J.; Pilon, G.; Dudonne, S.; Marette, A.; Jacques, H. Strawberry and cranberry polyphenols improve insulin sensitivity in insulin-resistant, non-diabetic adults: A parallel, double-blind, controlled and randomised clinical trial. Br. J. Nutr. 2017, 117, 519-531. [CrossRef]

20. Rohatgi, A.; Khera, A.; Berry, J.D.; Givens, E.G.; Ayers, C.R.; Wedin, K.E.; Neeland, I.J.; Yuhanna, I.S.; Rader, D.R.; de Lemos, J.A.; et al. HDL cholesterol efflux capacity and incident cardiovascular events. N. Engl. J. Med. 2014, 371, 2383-2393. [CrossRef] [PubMed]

21. Chobanian, A.V.; Bakris, G.L.; Black, H.R.; Cushman, W.C.; Green, L.A.; Izzo, J.L., Jr.; Jones, D.W.; Materson, B.J.; Oparil, S.; Wright, J.T., Jr.; et al. The Seventh Report of the Joint National Committee on Prevention, Detection, Evaluation, and Treatment of High Blood Pressure: The JNC 7 report. JAMA 2003, 289, 2560-2572. [CrossRef] [PubMed]

22. Richter, C.K.; Skulas-Ray, A.C.; Fleming, J.A.; Link, C.J.; Mukherjea, R.; Krul, E.S.; Kris-Etherton, P.M. Effects of isoflavonecontaining soya protein on ex vivo cholesterol efflux, vascular function and blood markers of CVD risk in adults with moderately elevated blood pressure: A dose-response randomised controlled trial. Br. J. Nutr. 2017, 117, 1403-1413. [CrossRef] [PubMed]

23. Chen, C.H.; Nevo, E.; Fetics, B.; Pak, P.H.; Yin, F.C.; Maughan, W.L.; Kass, D.A. Estimation of central aortic pressure waveform by mathematical transformation of radial tonometry pressure. Validation of generalized transfer function. Circulation 1997, 95, 1827-1836. [CrossRef]

24. Pauca, A.L.; O'Rourke, M.F.; Kon, N.D. Prospective evaluation of a method for estimating ascending aortic pressure from the radial artery pressure waveform. Hypertension 2001, 38, 932-937. [CrossRef]

25. Sharman, J.E.; Lim, R.; Qasem, A.M.; Coombes, J.S.; Burgess, M.I.; Franco, J.; Garrahy, P.; Wilkinson, I.B.; Marwick, T.H. Validation of a generalized transfer function to noninvasively derive central blood pressure during exercise. Hypertension 2006, 47, 1203-1208. [CrossRef] [PubMed]

26. Wilkinson, I.B.; MacCallum, H.; Flint, L.; Cockcroft, J.R.; Newby, D.E.; Webb, D.J. The influence of heart rate on augmentation index and central arterial pressure in humans. J. Physiol. 2000, 525, 263-270. [CrossRef] [PubMed]

27. Laurent, S.; Cockcroft, J.; Van Bortel, L.; Boutouyrie, P.; Giannattasio, C.; Hayoz, D.; Pannier, B.; Vlachopoulos, C.; Wilkinson, I.; Struijker-Boudier, H. Expert consensus document on arterial stiffness: Methodological issues and clinical applications. Eur. Heart J. 2006, 27, 2588-2605. [CrossRef]

28. Milne, G.L.; Sanchez, S.C.; Musiek, E.S.; Morrow, J.D. Quantification of F2-isoprostanes as a biomarker of oxidative stress. Nat. Protoc. 2007, 2, 221-226. [CrossRef]

29. Heffron, S.P.; Lin, B.X.; Parikh, M.; Scolaro, B.; Adelman, S.J.; Collins, H.L.; Berger, J.S.; Fisher, E.A. Changes in High-Density Lipoprotein Cholesterol Efflux Capacity After Bariatric Surgery Are Procedure Dependent. Arterioscler. Thromb. Vasc. Biol. 2018, 38, 245-254. [CrossRef] [PubMed]

30. Gille, A.; D'Andrea, D.; Tortorici, M.A.; Hartel, G.; Wright, S.D. CSL112 (Apolipoprotein A-I [Human]) Enhances Cholesterol Efflux Similarly in Healthy Individuals and Stable Atherosclerotic Disease Patients. Arterioscler. Thromb. Vasc. Biol. 2018, 38, 953-963. [CrossRef]

31. Skulas-Ray, A.C. Omega-3 fatty acids and inflammation: A perspective on the challenges of evaluating efficacy in clinical research. Prostaglandins Other Lipid Mediat. 2015, 116, 6390-6404. [CrossRef] [PubMed]

32. Harada, P.H.N.; Demler, O.V.; Dugani, S.B.; Akinkuolie, A.O.; Moorthy, M.V.; Ridker, P.M.; Cook, N.R.; Pradhan, A.D.; Mora, S. Lipoprotein insulin resistance score and risk of incident diabetes during extended follow-up of 20 years: The Women's Health Study. J. Clin. Lipidol. 2017, 11, 1257-1267. [CrossRef] [PubMed]

33. Shalaurova, I.; Connelly, M.A.; Garvey, W.T.; Otvos, J.D. Lipoprotein insulin resistance index: A lipoprotein particle-derived measure of insulin resistance. Metab. Syndr. Relat. Disord. 2014, 12, 422-429. [CrossRef]

34. Jennings, A.; Welch, A.A.; Fairweather-Tait, S.J.; Kay, C.; Minihane, A.M.; Chowienczyk, P.; Jiang, B.; Cecelja, M.; Spector, T.; Macgregor, A.; et al. Higher anthocyanin intake is associated with lower arterial stiffness and central blood pressure in women. Am. J. Clin. Nutr. 2012, 96, 781-788. [CrossRef]

35. Kowalska, K.; Olejnik, A.; Szwajgier, D.; Olkowicz, M. Inhibitory activity of chokeberry, bilberry, raspberry and cranberry polyphenol-rich extract towards adipogenesis and oxidative stress in differentiated 3T3-L1 adipose cells. PLoS ONE 2017, 12, e0188583. [CrossRef] 
36. Verdecchia, P. Prognostic value of ambulatory blood pressure: Current evidence and clinical implications. Hypertension 2000, 35, 844-851. [CrossRef]

37. Verdecchia, P.; Angeli, F.; Cavallini, C. Ambulatory blood pressure for cardiovascular risk stratification. Circulation 2007, 115, 2091-2093. [CrossRef]

38. Staessen, J.A.; Thijs, L.; Fagard, R.; O’Brien, E.T.; Clement, D.; de Leeuw, P.W.; Mancia, G.; Nachev, C.; Palatini, P.; Parati, G.; et al. Predicting cardiovascular risk using conventional vs ambulatory blood pressure in older patients with systolic hypertension. Systolic Hypertension in Europe Trial Investigators. JAMA 1999, 282, 539-546. [CrossRef]

39. Draijer, R.; de Graaf, Y.; Slettenaar, M.; de Groot, E.; Wright, C.I. Consumption of a polyphenol-rich grape-wine extract lowers ambulatory blood pressure in mildly hypertensive subjects. Nutrients 2015, 7, 3138-3153. [CrossRef] [PubMed]

40. Dohadwala, M.M.; Hamburg, N.M.; Holbrook, M.; Kim, B.H.; Duess, M.A.; Levit, A.; Titas, M.; Chung, W.B.; Vincent, F.B.; Caiano, T.L.; et al. Effects of Concord grape juice on ambulatory blood pressure in prehypertension and stage 1 hypertension. Am. J. Clin. Nutr. 2010, 92, 1052-1059. [CrossRef] [PubMed]

41. Ras, R.T.; Zock, P.L.; Zebregs, Y.E.; Johnston, N.R.; Webb, D.J.; Draijer, R. Effect of polyphenol-rich grape seed extract on ambulatory blood pressure in subjects with pre- and stage I hypertension. Br. J. Nutr. 2013, 110, 2234-2241. [CrossRef]

42. de Bock, M.; Derraik, J.G.; Brennan, C.M.; Biggs, J.B.; Morgan, P.E.; Hodgkinson, S.C.; Hofman, P.L.; Cutfield, W.S. Olive (Olea europaea L.) leaf polyphenols improve insulin sensitivity in middle-aged overweight men: A randomized, placebo-controlled, crossover trial. PLoS ONE 2013, 8, e57622. [CrossRef] [PubMed]

43. Habauzit, V.; Verny, M.A.; Milenkovic, D.; Barber-Chamoux, N.; Mazur, A.; Dubray, C.; Morand, C. Flavanones protect from arterial stiffness in postmenopausal women consuming grapefruit juice for 6 mo: A randomized, controlled, crossover trial. Am. J. Clin. Nutr. 2015, 102, 66-74. [CrossRef] [PubMed]

44. Keane, K.M.; George, T.W.; Constantinou, C.L.; Brown, M.A.; Clifford, T.; Howatson, G. Effects of Montmorency tart cherry (Prunus cerasus L.) consumption on vascular function in men with early hypertension. Am. J. Clin. Nutr. 2016, 103, 1531-1539. [CrossRef]

45. Curtis, P.J.; van der Velpen, V.; Berends, L.; Jennings, A.; Feelisch, M.; Umpleby, A.M.; Evans, M.; Fernandez, B.O.; Meiss, M.S.; Minnion, M.; et al. Blueberries improve biomarkers of cardiometabolic function in participants with metabolic syndrome-results from a 6-month, double-blind, randomized controlled trial. Am. J. Clin. Nutr. 2019, 109, 1535-1545. [CrossRef]

46. McAnulty, L.S.; Collier, S.R.; Landram, M.J.; Whittaker, D.S.; Isaacs, S.E.; Klemka, J.M.; Cheek, S.L.; Arms, J.C.; McAnulty, S.R. Six weeks daily ingestion of whole blueberry powder increases natural killer cell counts and reduces arterial stiffness in sedentary males and females. Nutr. Res. 2014, 34, 577-584. [CrossRef]

47. Asgary, S.; Sahebkar, A.; Afshani, M.R.; Keshvari, M.; Haghjooyjavanmard, S.; Rafieian-Kopaei, M. Clinical evaluation of blood pressure lowering, endothelial function improving, hypolipidemic and anti-inflammatory effects of pomegranate juice in hypertensive subjects. Phytother. Res. 2014, 28, 193-199. [CrossRef]

48. Lynn, A.; Hamadeh, H.; Leung, W.C.; Russell, J.M.; Barker, M.E. Effects of pomegranate juice supplementation on pulse wave velocity and blood pressure in healthy young and middle-aged men and women. Plant Foods Hum. Nutr. 2012, 67, 309-314. [CrossRef]

49. Tsang, C.; Smail, N.F.; Almoosawi, S.; McDougall, G.J.M.; Al-Dujaili, E.A.S. Antioxidant Rich Potato Improves Arterial Stiffness in Healthy Adults. Plant Foods Hum. Nutr. 2018, 73, 203-208. [CrossRef]

50. Appel, L.J.; Moore, T.J.; Obarzanek, E.; Vollmer, W.M.; Svetkey, L.P.; Sacks, F.M.; Bray, G.A.; Vogt, T.M.; Cutler, J.A.; Windhauser, M.M.; et al. A clinical trial of the effects of dietary patterns on blood pressure. DASH Collaborative Research Group. N. Engl. J. Med. 1997, 336, 1117-1124. [CrossRef] [PubMed]

51. McKay, D.L.; Chen, C.Y.; Zampariello, C.A.; Blumberg, J.B. Flavonoids and phenolic acids from cranberry juice are bioavailable and bioactive in healthy older adults. Food Chem. 2015, 168, 233-240. [CrossRef] [PubMed]

52. Rodriguez-Mateos, A.; Feliciano, R.P.; Boeres, A.; Weber, T.; Dos Santos, C.N.; Ventura, M.R.; Heiss, C. Cranberry (poly)phenol metabolites correlate with improvements in vascular function: A double-blind, randomized, controlled, dose-response, crossover study. Mol. Nutr. Food Res. 2016, 60, 2130-2140. [CrossRef]

53. Milbury, P.E.; Vita, J.A.; Blumberg, J.B. Anthocyanins are bioavailable in humans following an acute dose of cranberry juice. $J$. Nutr. 2010, 140, 1099-1104. [CrossRef] [PubMed]

54. Martini, D.; Marino, M.; Angelino, D.; Del Bo, C.; Del Rio, D.; Riso, P.; Porrini, M. Role of berries in vascular function: A systematic review of human intervention studies. Nutr. Rev. 2019, 78, 189-206. [CrossRef]

55. Kalt, W.; Blumberg, J.B.; McDonald, J.E.; Vinqvist-Tymchuk, M.R.; Fillmore, S.A.; Graf, B.A.; O'Leary, J.M.; Milbury, P.E. Identification of anthocyanins in the liver, eye, and brain of blueberry-fed pigs. J. Agric. Food Chem. 2008, 56, 705-712. [CrossRef] [PubMed]

56. Fairlie-Jones, L.; Davison, K.; Fromentin, E.; Hill, A.M. The Effect of Anthocyanin-Rich Foods or Extracts on Vascular Function in Adults: A Systematic Review and Meta-Analysis of Randomised Controlled Trials. Nutrients 2017, 9, 908. [CrossRef] [PubMed]

57. Huang, H.; Chen, G.; Liao, D.; Zhu, Y.; Xue, X. Effects of Berries Consumption on Cardiovascular Risk Factors: A Meta-analysis with Trial Sequential Analysis of Randomized Controlled Trials. Sci. Rep. 2016, 6, 23625. [CrossRef] [PubMed]

58. Allaire, J.; Vors, C.; Couture, P.; Lamarche, B. LDL particle number and size and cardiovascular risk: Anything new under the sun? Curr. Opin. Lipidol. 2017, 28, 261-266. [CrossRef] [PubMed]

59. Krauss, R.M. Lipoprotein subfractions and cardiovascular disease risk. Curr. Opin. Lipidol. 2010, 21, 305-311. [CrossRef] 
60. Sacks, F.M.; Campos, H. Clinical review 163: Cardiovascular endocrinology: Low-density lipoprotein size and cardiovascular disease: A reappraisal. J. Clin. Endocrinol. Metab. 2003, 88, 4525-4532. [CrossRef]

61. Shiffman, D.; Louie, J.Z.; Caulfield, M.P.; Nilsson, P.M.; Devlin, J.J.; Melander, O. LDL subfractions are associated with incident cardiovascular disease in the Malmo Prevention Project Study. Atherosclerosis 2017, 263, 287-292. [CrossRef]

62. Qiu, C.; Zhao, X.; Zhou, Q.; Zhang, Z. High-density lipoprotein cholesterol efflux capacity is inversely associated with cardiovascular risk: A systematic review and meta-analysis. Lipids Health Disord. 2017, 16, 212. [CrossRef]

63. Khera, A.V.; Demler, O.V.; Adelman, S.J.; Collins, H.L.; Glynn, R.J.; Ridker, P.M.; Rader, D.J.; and Mora, S. Cholesterol Efflux Capacity, High-Density Lipoprotein Particle Number, and Incident Cardiovascular Events: An Analysis From the JUPITER Trial (Justification for the Use of Statins in Prevention: An Intervention Trial Evaluating Rosuvastatin). Circulation 2017, 135, 2494-2504. [CrossRef]

64. Ruel, G.; Pomerleau, S.; Couture, P.; Lemieux, S.; Lamarche, B.; Couillard, C. Favourable impact of low-calorie cranberry juice consumption on plasma HDL-cholesterol concentrations in men. Br. J. Nutr. 2006, 96, 357-364. [CrossRef]

65. Eftekhari, M.H.; Allaei, M.; Khosropanah, S.; Rajaeifard, A.; Sohrabi, Z. Cranberry Supplement and Metabolic Risk Factors in Obese and Overweight Females. Jentashapir J. Health Res. 2016, 7, e37255. [CrossRef]

66. Zhu, Y.; Huang, X.; Zhang, Y.; Wang, Y.; Liu, Y.; Sun, R.; Xia, M. Anthocyanin supplementation improves HDL-associated paraoxonase 1 activity and enhances cholesterol efflux capacity in subjects with hypercholesterolemia. J. Clin. Endocrinol. Metab. 2014, 99, 561-569. [CrossRef] [PubMed]

67. Berryman, C.E.; Fleming, J.A.; Kris-Etherton, P.M. Inclusion of Almonds in a Cholesterol-Lowering Diet Improves Plasma HDL Subspecies and Cholesterol Efflux to Serum in Normal-Weight Individuals with Elevated LDL Cholesterol. J. Nutr. 2017, 147, 1517-1523. [CrossRef] [PubMed]

68. Ridker, P.M. Clinical application of C-reactive protein for cardiovascular disease detection and prevention. Circulation 2003, 107, 363-369. [CrossRef]

69. Whelton, P.K.; Carey, R.M.; Aronow, W.S.; Casey, D.E.; Collins, K.J.; Himmelfarb, C.D.; DePalma, S.M.; Gidding, S.; Jamerson, K.A.; Jones, D.W. 2017 ACC/AHA/AAPA/ABC/ACPM/AGS/APhA/ASH/ASPC/NMA/PCNA guideline for the prevention, detection, evaluation, and management of high blood pressure in adults: A report of the American College of Cardiology/American Heart Association Task Force on Clinical Practice Guidelines. J. Am. Coll. Cardiol. 2018, 71, e127-e248.

70. Vinson, J.A.; Bose, P.; Proch, J.; Al Kharrat, H.; Samman, N. Cranberries and cranberry products: Powerful in vitro, ex vivo, and in vivo sources of antioxidants. J. Agric. Food Chem. 2008, 56, 5884-5891. [CrossRef] [PubMed] 